\title{
MULTISCALE COARSE SPACES FOR OVERLAPPING SCHWARZ METHODS BASED ON THE ACMS SPACE IN 2D*
}

\author{
ALEXANDER HEINLEIN $^{\dagger}$, AXEL KLAWONN $^{\dagger}$, JASCHA KNEPPER $^{\dagger}$, AND OLIVER RHEINBACH ${ }^{\ddagger}$
}

\begin{abstract}
Two-level overlapping Schwarz domain decomposition methods for second-order elliptic problems in two dimensions are proposed using coarse spaces constructed from the Approximate Component Mode Synthesis (ACMS) multiscale discretization approach. These coarse spaces are based on eigenvalue problems using Schur complements on subdomain edges. It is then shown that the convergence of the resulting preconditioned Krylov method can be controlled by a user-specified tolerance and thus can be made independent of heterogeneities in the coefficient of the partial differential equation. The relations of this new approach to other known adaptive coarse space approaches for overlapping Schwarz methods are also discussed. Compared to one of the competing adaptive approaches, the new coarse space can be significantly smaller. Compared to other competing approaches, the eigenvalue problems are significantly cheaper to solve, i.e., the dimension of the eigenvalue problems is minimal among the competing adaptive approaches under consideration. Our local eigenvalue problems can be solved using one iteration of LobPCG for essentially the same cost as a Cholesky-decomposition of a Schur complement on a subdomain edge.
\end{abstract}

Key words. domain decomposition, multiscale, approximate component mode synthesis (ACMS), scientific software, parallel, overlapping Schwarz

AMS subject classifications. 65F08, 65F10, 65N55, 68W10, 74S30

1. Introduction. We consider coarse spaces for two-level overlapping Schwarz methods [40] based on variations of the Approximate Component Mode Synthesis (ACMS) special finite element method, which was introduced in [25] for the discretization of second-order elliptic problems with highly varying coefficients. For our new two-level overlapping Schwarz method, we obtain a condition number bound which is independent of large variations in the coefficient; see Section 6 for the condition number bound and Section 7 for corresponding numerical results.

The ACMS discretization method builds on the traditional Component Mode Synthesis (CMS) method [7, 27] and uses eigenvectors from local discrete eigenvalue problems to construct the approximation space. A discretization error estimate and an a posteriori error indicator are available [24] for ACMS as well as a parallel implementation [22]. The improved approximation properties for heterogeneous problems, compared to standard FEM spaces, rely on the adaptation of the basis functions to the heterogeneities, i.e., the basis functions are computed in a first (off line) step. They are used in the later (on line) simulations, which can be performed on a relatively coarse mesh. However, for large problems, this mesh may still require parallel solution methods, and in [22], it has been shown that parallel domain decomposition solvers can robustly solve the discretized ACMS (on line) problems.

Many multiscale discretization methods have been proposed in the past, among them are Multiscale Finite Element Methods (MsFEM) [15, 26], Heterogeneous Multiscale Finite Element Methods (HMM) [13, 14], adaptive multiscale methods [35], and Generalized Finite Element Methods (GFEM) [2,3]; see also the references therein.

Different exotic coarse spaces for overlapping Schwarz methods are also known, e.g., energy minimizing coarse spaces $[1,5,6,8,9,10,20,23]$, where $[1,5,6]$ use coarse spaces

\footnotetext{
* Received March 8, 2016. Accepted April 8, 2018. Published online on May 21, 2018. Recommended by Martin Gander.

${ }^{\dagger}$ Mathematisches Institut, Universität zu Köln, Weyertal 86-90, 50931 Köln, Germany

(\{alexander.heinlein, axel.klawonn, jascha.knepper\}@uni-koeln.de).

${ }^{\ddagger}$ Institut für Numerische Mathematik und Optimierung, Fakultät für Mathematik und Informatik, Technische Universität Bergakademie Freiberg, Akademiestr. 6, 09599 Freiberg

(oliver.rheinbach@math.tu-freiberg.de).
} 
TABLE 2.1

Reference table for some bilinear forms, semi-norms, and norms used throughout this paper; see the respective reference for an exact definition. The upper part lists those related corresponding to the $a(\cdot, \cdot)$ bilinear form, whereas the lower part lists those related to the scaled $L^{2}$-inner product.

\begin{tabular}{ll|l}
$a_{\Omega}(u, v)$ & $:=\int_{\Omega} A(x)(\nabla u(x))^{T} \nabla v(x) d x$ & $(2.3)$ \\
$|u|_{a, \Omega}^{2}$ & $:=a_{\Omega}(u, u)$ & $(2.4)$ \\
$a_{*, e, \eta}(u, v)$ & $:=a_{\eta}\left(\mathcal{H}_{e \rightarrow \eta}(u), \mathcal{H}_{e \rightarrow \eta}(v)\right)$ & $(4.11)$ \\
$|u|_{a, *, e, \eta}^{2}$ & $:=a_{*, e, \eta}(u, u)$ & $(5.12)$ \\
$\bar{a}_{e_{i j}}(u, v)$ & $:=\left(A_{e_{i j}, \max }(x) D_{x^{t}} u, D_{x^{t}} v\right)$ & $(4.1)$ \\
\hline$b_{e_{i j}}(u, v)$ & $:=\frac{1}{h}\left(A_{e_{i j}, \max } u, v\right)_{L^{2}\left(e_{i j}\right)}$ & \\
$\tilde{b}_{e_{i j}}(u, v)$ & $:=\frac{1}{h^{2}}\left(A w_{e_{i j}}(u), w_{e_{i j}}(v)\right)_{L^{2}\left(\Omega_{e_{i j}}\right)}$ & \\
$\|u\|_{\tilde{b}, e_{i j}}^{2}$ & $:=\tilde{b}_{e_{i j}}(u, u)$ & \\
$\bar{b}_{e_{i j}}(u, v)$ & $:=h^{-1} \sum_{x_{k} \in e_{i j}} \beta_{k} u\left(x_{k}\right) v\left(x_{k}\right)$ &
\end{tabular}

built on MsFEM basis functions. Adaptive coarse spaces from eigenvalue problems for overlapping Schwarz methods were already proposed in $[16,17]$ considering unions of neighboring subdomains. Their idea was to replace a Poincaré inequality by a computable bound from a discrete eigenvalue problem involving a Neumann matrix and a mass matrix. In [38], Spillane et al. introduced the GenEO coarse space for overlapping Schwarz methods. This method uses the bilinear form on both sides of the generalized eigenvalue problems. By elimination, the generalized eigenvalue problems can be reduced to the overlap of the subdomains, resulting in smaller eigenvalue problems. This helped to make adaptive overlapping Schwarz methods practical.

The size of the eigenvalue problems is further reduced in the more recent methods which are the focus of this paper. Here, (generalized) eigenvalue problems on the subdomain boundary or on edges are solved. We will briefly review ACMS as a discretization method before we introduce the ACMS-based coarse space for overlapping Schwarz methods using eigenvalue problems on edges. We will also discuss two approaches closely related to ours, i.e., the method introduced in [11], where eigenvalue problems on the boundaries of the overlapping subdomains are used, and the very recent method introduced in [19] also using edges. The methods [16, 17], [11], and [19] are described in Section 5.1, Section 5.2, and Section 5.3, respectively, in order to illustrate differences and relations to our approach. Let us note that, compared to [11, 16, 17], the eigenvalue problems in our approach are more local, i.e., defined on edges. The eigenvalue problems used in [19] are also defined on edges and are typically computationally slightly cheaper than ours (if the standard, non-economic variant of our approach is used), but our new ACMS coarse space can be significantly smaller in certain cases; see Section 7. For an overview of the bilinear forms, semi-norms, and norms defined and used in this paper, we refer to Table 2.1.

Adaptive coarse space approaches for nonoverlapping domain decomposition methods are, of course, also related, cf., e.g., [4, 28, 29, 30, 31, 33, 34, 36, 37, 39], but are outside the scope of this paper. For further references to the literature, we refer to the references listed in the aforementioned publications.

2. ACMS special finite element method. We consider problems of the form

$$
\begin{aligned}
-\nabla \cdot(A(x) \nabla u(x)) & =f(x) & & \text { in } \Omega \subset \mathbb{R}^{2}, \\
u & =0 & & \text { on } \partial \Omega,
\end{aligned}
$$


where the scalar coefficient function $A: \mathbb{R}^{2} \rightarrow \mathbb{R}$ is highly heterogeneous, possibly with high jumps. The model problem (2.1) can be transformed into the variational formulation: find $u \in H_{0}^{1}(\Omega)$ such that

$$
a_{\Omega}(u, v)=L(v) \quad \forall v \in H_{0}^{1}(\Omega)
$$

with the bilinear form and the linear functional

$$
a_{\Omega}(u, v):=\int_{\Omega} A(x)(\nabla u(x))^{T} \nabla v(x) d x \quad \text { and } \quad L(v):=\int_{\Omega} f(x) v(x) d x,
$$

respectively, where $f \in L^{2}(\Omega)$. In addition to that, we denote the semi-norm corresponding to the bilinear form $a_{\Omega}(\cdot, \cdot)$ as

$$
|u|_{a, \Omega}^{2}:=a_{\Omega}(u, u) .
$$

We assume that the coefficient function $A(x)$ satisfies

$$
0<A_{\min } \leq A(x) \leq A_{\max } \quad \forall x \in \bar{\Omega} .
$$

We consider a family $\left(\tau_{H}\right)_{H}$ of conforming triangulations of $\Omega$ into triangles or convex quadrilaterals, where each triangulation introduces an interface

$$
\Gamma=\left(\bigcup_{T \in \tau_{H}} \partial T\right) \backslash \partial \Omega .
$$

The decomposition

$$
H_{0}^{1}(\Omega)=\left(\bigoplus_{T \in \tau_{H}} V_{T}\right) \oplus V_{\Gamma}
$$

is orthogonal with respect to $a_{\Omega}(\cdot, \cdot)$ and is standard in domain decomposition theory. However, here, the decomposition $\left(\tau_{H}\right)_{H}$ of $\Omega$ corresponds to a triangulation by ACMS elements with typical diameter $H$. Only later, when we use ACMS as a coarse space for overlapping Schwarz methods, this decomposition will correspond to a nonoverlapping domain decomposition with typical diameter $H$. Note that this domain decomposition may then also be irregular.

We have

$$
V_{T}=\left\{v \in H_{0}^{1}(\Omega):\left.v\right|_{T} \in H_{0}^{1}(T) \text { and }\left.v\right|_{\Omega \backslash \bar{T}}=0\right\} \subset H_{0}^{1}(\Omega)
$$

for all $T \in \tau_{H}$ and

$$
V_{\Gamma}=\left\{\mathcal{H}_{\Gamma \rightarrow \Omega}(\tau) \in H_{0}^{1}(\Omega): \tau \in H^{1 / 2}(\Gamma)\right\} \subset H_{0}^{1}(\Omega),
$$

where $\mathcal{H}_{\Gamma \rightarrow \Omega}$ corresponds to the harmonic extension operator, i.e., to the solution of

$$
\begin{array}{rlrl}
-\nabla \cdot\left(A(x) \nabla \mathcal{H}_{\Gamma \rightarrow \Omega}(\tau)\right) & =0 & & \text { in } T, \forall T \in \tau_{H}, \\
\mathcal{H}_{\Gamma \rightarrow \Omega}(\tau)=\tau & & \text { on } \Gamma, \\
\mathcal{H}_{\Gamma \rightarrow \Omega}(\tau)=0 & & \text { on } \partial \Omega .
\end{array}
$$




\section{ETNA}

Kent State University and

Johann Radon Institute (RICAM)

Based on decomposition (2.5), problem (2.2) can equivalently be written as: find $u_{T} \in V_{T}$ and $u_{\Gamma} \in V_{\Gamma}$ such that

$$
\begin{aligned}
a_{\Omega}\left(u_{T}, v_{T}\right)=L\left(v_{T}\right) & & \forall T \in \tau_{H}, \forall v_{T} \in V_{T}, \\
a_{\Omega}\left(u_{\Gamma}, v_{\Gamma}\right)=L\left(v_{\Gamma}\right) & & \forall v_{\Gamma} \in V_{\Gamma},
\end{aligned}
$$

where the solution of (2.2) is then given by

$$
u=\sum_{T \in \tau_{H}} u_{T}+u_{\Gamma} \quad \in H_{0}^{1}(\Omega) .
$$

In the CMS method [7, 27], eigenfunctions are used as basis functions to solve the problems given in (2.6); see also Kolmogoroff's $n$-width from approximation theory, which explains the approximation properties of eigenfunctions. The ACMS finite element space, which was introduced by Hetmaniuk and Lehoucq in [25], is designed as an approximation of the CMS finite element space where only basis functions with local support are employed. This applies, in particular, to the functions in $V_{\Gamma}$, which have global support, whereas the functions in $V_{T}$ are already local in the CMS method.

We employ, in total, three different types of basis functions in the ACMS method which we refer to as vertex-specific, edge-based, and fixed-interface basis functions.

A vertex-specific basis function which corresponds to a vertex $P$ of the triangulation $\tau_{H}$, is given by the boundary value problem

$$
\begin{aligned}
-\nabla \cdot\left(A(x) \nabla \varphi_{P}\right) & =0 & & \text { in } T, \forall T \in \tau_{H}, \\
\varphi_{P} & =0 & & \text { on } \partial \Omega, \\
\varphi_{P}\left(P^{\prime}\right) & =\delta_{P, P^{\prime}} & & \forall \text { vertices } P^{\prime},
\end{aligned}
$$

where $\delta_{P, P^{\prime}}$ is the Kronecker delta function. These functions originate from the Multiscale Finite Element Method (MsFEM) [15, 26], and we refer to them also as MsFEM basis functions. Note that from (2.7), the basis function $\varphi_{P}$ is not yet well defined since the definition of the values at the edges is missing.

These vertex-specific basis functions are harmonic extensions of trace functions defined on $\Gamma$, and thus $\varphi_{P} \in V_{\Gamma}$. The trace values of $\varphi_{P}$ on $\Gamma$ can, e.g., be chosen to be linear between the vertices or discrete harmonic in one dimension. Another possible way to define the edge values of the MsFEM basis functions is given in (4.4) in Section 4.2. In the latter case, on each edge $e \subset \Gamma$, we choose them to be the solution of

$$
\begin{aligned}
\frac{\partial}{\partial \tau}\left\langle A_{e, \max }(x) \tau, \nabla \varphi_{P}(x)\right\rangle & =0 & & \text { on } e, \\
\varphi_{P}\left(P^{\prime}\right) & =\delta_{P, P^{\prime}} & & \text { on } \Gamma,
\end{aligned}
$$

where $\tau$ denotes the tangential vector of the edge with $\|\tau\|=\sqrt{\langle\tau, \tau\rangle}=1$ and $\langle\cdot, \cdot\rangle$ is the standard Euclidean inner product. Here, we use for an edge $\bar{e}_{i j}=\bar{T}_{i} \cap \bar{T}_{j}$ with $T_{i}, T_{j} \in \tau_{H}$, $i \neq j$,

$$
A_{e_{i j}, \max }(x):=\max \left\{\lim _{y_{i} \in \Omega_{i} \rightarrow x} A\left(y_{i}\right), \lim _{y_{j} \in \Omega_{j} \rightarrow x} A\left(y_{j}\right)\right\}
$$

instead of $A$ in case of discontinuities across the edge; cf., e.g., the coefficient function in Figure 3.1. 
For any open edge $e \subset \Gamma$, we define the edge-based basis function by the corresponding eigenvalue problem in the space of harmonic extensions: find $\left(\tau_{*, e}, \lambda_{*, e}\right) \in H_{00}^{1 / 2}(e) \times \mathbb{R}$ such that

$$
a_{\Omega}\left(\mathcal{H}_{\Gamma \rightarrow \Omega}\left(\tilde{\tau}_{*, e}\right), \mathcal{H}_{\Gamma \rightarrow \Omega}(\tilde{\theta})\right)=\lambda_{*, e}\left(\tau_{*, e}, \theta\right)_{L^{2}(e)} \quad \forall \theta \in H_{00}^{1 / 2}(e)
$$

with $\tilde{\tau}_{*, e}$ and $\tilde{\theta}$ being the trivial extension of $\tau_{*, e}$ and $\theta$, respectively, by zero to $\Gamma \backslash e$. In order to approximate the solution of the local problems in (2.6), fixed-interface eigenfunctions with

$$
a_{\Omega}\left(z_{*, T}, v\right)=\lambda_{*, T}\left(z_{*, T}, v\right)_{L^{2}(\Omega)} \quad \forall v \in V_{T}
$$

on each of the $T \in \tau_{H}$ are employed. The eigenvalues $\left\{\lambda_{i, T}\right\}_{i=1}^{\infty}$ and $\left\{\lambda_{i, e}\right\}_{i=1}^{\infty}$ are assumed to be ordered non-decreasingly and the corresponding eigenmodes accordingly. The eigenmodes $z_{1, T}, z_{2, T}, \ldots$ and $\tau_{1, e}, \tau_{2, e}, \ldots$ form orthonormal bases for the $L^{2}$-inner product of $V_{T}$ and of $V_{\Gamma}$ on the element $T$ and on the edge $e$, respectively.

The finite element space of the ACMS special finite element method is then given by

$$
\begin{aligned}
V_{\mathrm{ACMS}}= & \left(\bigoplus_{T \in \tau_{H}} \operatorname{span}\left\{z_{i, T}: 1 \leq i \leq I_{T}\right\}\right) \\
& \oplus\left(\bigoplus_{\substack{P \in \Gamma \\
P \text { vertex }}} \operatorname{span}\left\{\varphi_{P}\right\}\right) \\
& \oplus\left(\bigoplus_{\substack{e \subset \Gamma \\
e \text { edge }}} \operatorname{span}\left\{\mathcal{H}_{\Gamma \rightarrow \Omega}\left(\tilde{\tau}_{i, e}\right): 1 \leq i \leq I_{e}\right\}\right)
\end{aligned}
$$

with positive integers $I_{T}$, for all $T \in \tau_{H}$, and $I_{e}$, for all edges $e \subset \Gamma$, corresponding to the number of eigenmodes used as basis functions in each element and on each edge, respectively.

In practice, the ACMS basis functions are not computed explicitly, but they are replaced by their approximation on a submesh; cf. [22, 25]. This submesh is also denoted as the ACMS fine mesh $\tau_{h}$, and its finite elements have a typical diameter of $h$. Next, in the overlapping Schwarz method with the ACMS coarse space, the standard finite element mesh will correspond to the ACMS fine mesh, and the ACMS elements $T \in \tau_{H}$ with a typical diameter of $H$ will correspond to the nonoverlapping domain decomposition underlying the overlapping Schwarz method.

3. Two-level overlapping Schwarz methods. Let

$$
K u=f
$$

be the discretization of the problem (2.2) by piecewise linear or bilinear finite elements on a family of triangulations $\left(\tau_{h}\right)_{h}, K$ the stiffness matrix, $f$ the right-hand side, and $u$ the discrete solution vector in the finite element space $V:=V^{h}(\Omega)$. Furthermore, let $\left\{\Omega_{i}\right\}_{i=1}^{N}$ be a nonoverlapping domain decomposition of $\Omega$ with the interface

$$
\Gamma=\bigcup_{i=1}^{N} \partial \Omega_{i} \backslash \partial \Omega .
$$

The typical diameter of the subdomains is $H$. 
TABLE 3.1

Iteration counts and condition numbers for PCG with a two-level Schwarz preconditioner using Q1 (piecewise bilinear) or MsFEM coarse basis functions; $1 / H=4, H / h=16$, and $\delta=2 h$; relative stopping criterion $\left\|r^{(k)}\right\|_{2} /\left\|r^{(0)}\right\|_{2}<10^{-8}$; for the coefficient distribution, see Figure 3.1.

\begin{tabular}{l|rr}
$V_{0}$ & it. & $\kappa$ \\
\hline$V_{\mathrm{Q} 1}$ & 98 & $8.87 \cdot 10^{5}$ \\
$V_{\mathrm{MsFEM}}$ & 21 & 5.56
\end{tabular}

The set $\left\{\Omega_{i}^{\prime}\right\}_{i=1}^{N}$ is a corresponding overlapping decomposition of $\Omega$ with overlap $\delta$. In the preconditioning context, we assume that the coefficient function $A$ is constant on each finite element. We define as $R_{i}: V \rightarrow V_{i}:=V^{h}\left(\Omega_{i}^{\prime}\right), i=1, \ldots, N$, the restriction to the local finite element space on the overlapping subdomain $\Omega_{i}^{\prime}$, and $R_{i}^{T}$ is the corresponding prolongation to $V^{h}(\Omega)$. In addition, let $V_{0}$ be a global coarse space. We use exact local solvers, and therefore the local bilinear forms on the subspaces are given by

$$
\tilde{a}_{i}\left(u_{i}, v_{i}\right)=a_{\Omega}\left(R_{i}^{T} u_{i}, R_{i}^{T} v_{i}\right) \quad \forall u_{i}, v_{i} \in V_{i}
$$

and the operators $\tilde{P}_{i}: V \rightarrow V_{i}$ by

$$
\tilde{a}_{i}\left(\tilde{P}_{i} u, v_{i}\right)=a_{\Omega}\left(u, R_{i}^{T} v_{i}\right) \quad \forall v_{i} \in V_{i}
$$

for $i=1, \ldots, N$; cf., e.g., [40]. Defining $P_{i}:=R_{i}^{T} \tilde{P}_{i}: V \rightarrow R_{i}^{T} V_{i} \subset V$, the two-level Schwarz operator is given by

$$
P_{\mathrm{OS}-2}=\sum_{i=0}^{N} P_{i},
$$

and the two-level overlapping Schwarz preconditioner in matrix form can be written as

$$
M_{\mathrm{OS}-2}^{-1}=\underbrace{R_{0}^{T} K_{0}^{-1} R_{0}}_{\text {coarse level }}+\underbrace{\sum_{i=1}^{N} R_{i}^{T} K_{i}^{-1} R_{i}}_{\text {first level }} .
$$

Here, the local and coarse stiffness matrices $K_{i}$ are given by

$$
K_{i}=R_{i} K R_{i}^{T},
$$

for $i=0, \ldots, N$. Note that the coarse operator $K_{0}$ is formed by a Galerkin product instead of using a discretization on a coarse grid.

The condition number of the standard two-level Schwarz preconditioner for the problem (2.2), where standard Lagrange basis functions are used for the discretization of the coarse level, depends on the contrast of the coefficient function $A$, i.e.,

$$
\kappa\left(M_{\mathrm{OS}-2}^{-1} K\right) \leq C \max _{T \in \tau_{H}} \max _{x, y \in \omega_{T}}\left(\frac{A(x)}{A(y)}\right)\left(1+\frac{H}{\delta}\right) .
$$

Here, $\omega_{T}$ corresponds to the union of all coarse mesh elements which touch a coarse mesh element $T$. This bound can be sharpened, but a dependence on the coefficient contrast remains; see [20]. 


\section{ETNA}

Kent State University and

Johann Radon Institute (RICAM)

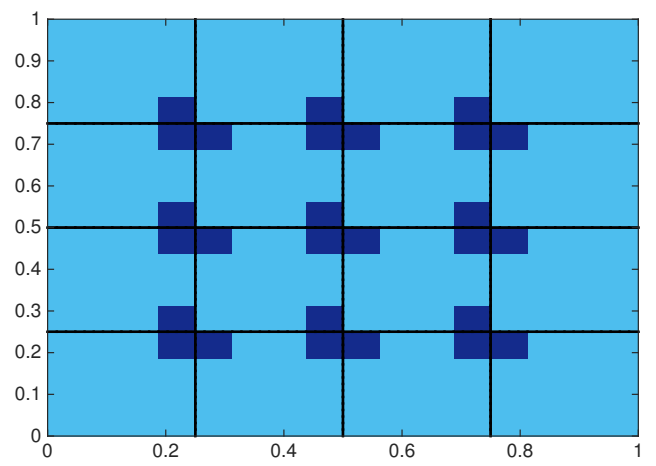

FIG. 3.1. A discontinuous coefficient function A with inclusion at the vertices of the decomposition. The light blue color corresponds to a coefficient of 1.0 and the dark blue color to a coefficient of $10^{8}, 1 / \mathrm{H}=4$.

3.1. The MsFEM coarse space. As proposed by Aarnes and Hou [1] as well as by Buck, Iliev, and Andrä [5, 6], MsFEM basis functions can be used in the coarse space of an overlapping Schwarz preconditioner to enhance the performance in the presence of rough coefficients.

Considering the overlapping Schwarz method and the underlying domain decomposition into nonoverlapping subdomains $\Omega_{1}, \ldots, \Omega_{N}$ of typical diameter $H$, we identify each $\Omega_{i}$ with an element $T \in \tau_{H}$ from Section 2. The MsFEM basis functions $\varphi_{P}$, as defined in (2.7), are then discretized in the spaces $V^{h}\left(\Omega_{i}\right)$, i.e., on a fine triangulation of $\Omega_{i}$ into finite elements of typical diameter $h$; we also refer to the fine triangulation as the fine mesh.

In particular, the discretized basis functions, which we will also denote as $\varphi_{P}$, are employed to build the coarse MsFEM space

$$
V_{\mathrm{MsFEM}-\mathrm{O}}:=\left(\bigoplus_{\substack{P \in \Gamma \\ P \text { vertex }}} \operatorname{span}\left\{\varphi_{P}\right\}\right) ;
$$

cf. [25] for the notation "MsFEM-O". Since these functions are thus defined as discrete harmonic extensions, they can be computed also for unstructured domain decompositions (as in the GDSW preconditioner $[8,9,23]$ ) without the need for an additional coarse triangulation. This is an advantage over the use of standard Lagrange coarse basis functions. The GDSW preconditioner is a two-level overlapping Schwarz preconditioner with energy minimizing coarse space functions, i.e., it uses discrete harmonic coarse space functions as well. MsFEM basis functions can also improve significantly the robustness of the overlapping Schwarz preconditioner for coefficient functions of a certain (simple) type; cf., e.g., Figure 3.1 and the results in Table 3.1.

Note that, for a constant coefficient function $A$ and a structured domain decomposition into triangles or quadrilaterals, the MsFEM coarse space corresponds to standard piecewise linear or bilinear coarse basis functions, respectively.

4. Coarse spaces based on the ACMS space. In adaptive coarse spaces, generalized eigenvalue problems are typically used in order to obtain condition number estimates that are independent of the contrast of the coefficient function. Thus, it seems natural to use the ACMS edge-based basis functions as coarse basis functions in a two-level Schwarz method for heterogeneous problems; these ACMS edge functions are computed from local generalized eigenvalue problems (2.10) and contribute to the excellent approximation properties of the 
ACMS discretization for second-order elliptic PDEs with highly heterogeneous coefficients. We will observe, after applying some modifications to the eigenvalue problems, that we can construct a coarse space for overlapping Schwarz methods which is robust with respect to the contrast of the coefficient function; cf. Section 6 for a proof and Section 7 for supporting numerical results.

As in the two-level Schwarz preconditioner with an MsFEM coarse space (cf. Section 3.1), let us consider our overlapping Schwarz method using the underlying domain decomposition into nonoverlapping subdomains $\Omega_{1}, \ldots, \Omega_{N}$ of typical diameter $H$. In order to define our ACMS coarse space for the overlapping Schwarz method, we again identify each $\Omega_{i}$ with an ACMS element $T$; cf. Section 2. The triangulation of $\Omega_{i}$ into finite elements of typical diameter $h$ then represents the ACMS fine mesh $\tau_{h}$; for simplicity, we assume quasi-uniformity of the fine mesh.

4.1. Coarse space with Dirichlet boundary conditions. We propose a coarse space for overlapping Schwarz methods which is based on the construction of the ACMS finite element space. In particular, we use the MsFEM basis functions, i.e., the vertex-specific basis function (see (2.7)) and a modified version of the edge-based coupling eigenmodes (see (2.10)), where we replace the $L^{2}$-inner product on the right-hand side of (2.10) by a scaled version. Therefore, for an edge $\bar{e}_{i j}=\bar{\Omega}_{i} \cap \bar{\Omega}_{j}$, we define the bilinear form

$$
b_{e_{i j}}(u, v):=\frac{1}{h}\left(A_{e_{i j}, \max } u, v\right)_{L^{2}\left(e_{i j}\right)},
$$

where $A_{e_{i j} \text {, max }}$ is defined as in (2.9). Using this bilinear form, we obtain a discrete eigenvalue problem, which is a modification of (2.10): find

$$
\tau_{*, e_{i j}} \in V_{0}^{h}\left(e_{i j}\right):=\left\{\left.v\right|_{e_{i j}}: v \in V, v=0 \text { on } \partial e_{i j}\right\},
$$

such that

$$
a_{\Omega}\left(\mathcal{H}_{\Gamma \rightarrow \Omega}\left(\tilde{\tau}_{*, e_{i j}}\right), \mathcal{H}_{\Gamma \rightarrow \Omega}(\tilde{\theta})\right)=\lambda_{*, e_{i j}} b_{e_{i j}}\left(\tau_{*, e_{i j}}, \theta\right) \quad \forall \theta \in V_{0}^{h}\left(e_{i j}\right) .
$$

Again, the tilde corresponds to the extensions of $\tau_{*, e_{i j}}$ and $\theta$ by zero to $\Gamma \backslash e_{i j}$. Let the eigenvalues be sorted in non-decreasing order, i.e., $\lambda_{1, e_{i j}} \leq \lambda_{2, e_{i j}} \leq \ldots \leq \lambda_{m}$, and the eigenmodes accordingly, where $m=\operatorname{dim}\left(V_{0}^{h}\left(e_{i j}\right)\right)$. We select all eigenmodes $\tau_{*, e_{i j}}$ where the eigenvalues are below a certain threshold $t o l$, i.e., $\lambda_{*, e_{i j}} \leq t o l$. Then, the coarse basis functions corresponding to the edge $e_{i j}$ are the discrete harmonic extensions of the selected $\tilde{\tau}_{*, e_{i j}}$ to the interior degrees of freedom, i.e., the solutions $v_{*, e_{i j}}$ of

$$
\begin{aligned}
a_{\Omega_{l}}\left(v_{*, e_{i j}}, v\right) & =0 & & \forall v \in V_{0}^{h}\left(\Omega_{l}\right), l=1, \ldots, N, \\
v_{*, e_{i j}} & =\tau_{*, e_{i j}} & & \text { on } e_{i j}, \\
v_{*, e_{i j}} & =0 & & \text { on } \Gamma \backslash e_{i j},
\end{aligned}
$$

where $V_{0}^{h}\left(\Omega_{i}\right):=\left\{v: v \in V, v=0\right.$ in $\left.\Omega \backslash \Omega_{i}\right\}$.

The coarse space based on the ACMS finite element method is then given by

$$
V_{\mathrm{ACMS}-\mathrm{D}}^{\text {tol }}=V_{\mathrm{MsFEM}-\mathrm{O}} \oplus\left(\bigoplus_{\substack{e \subset \Gamma \\ e \text { edge }}} \operatorname{span}\left\{v_{k, e}: \lambda_{k, e} \leq t o l\right\}\right),
$$

where $V_{\mathrm{MsFEM}-\mathrm{O}}$ is the MsFEM coarse space, which is identical to the space spanned by the discretized vertex-based ACMS basis functions; $\mathrm{cf}$ (2.11). 


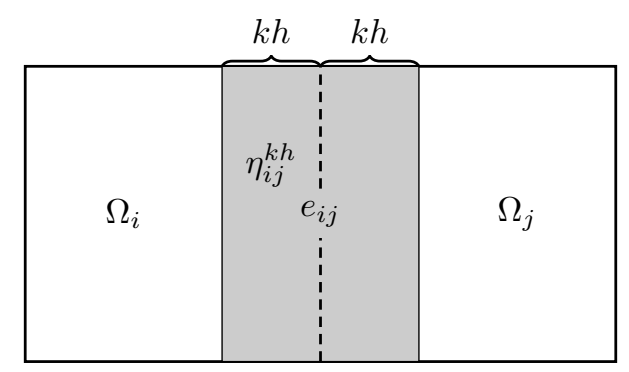

FIG. 4.1. Graphical representation of the slab $\eta_{i j}^{k h}$ corresponding to the edge $e_{i j}$. We denote the methods which construct the eigenvalue problems using functions supported on a slab as "economic".

As can be observed in Section 7, this coarse space is robust for many coefficient distributions with jumping coefficients, while the eigenvalue problems are defined on the open edges and are thus of comparably modest size.

Here, we enforce Dirichlet boundary conditions on the whole interface for the discrete harmonic extensions on the left-hand side of the generalized eigenvalue problem (4.2). However, for our proof, a different choice of the boundary conditions on the left-hand side of (4.2) will be beneficial; see the subsequent Section 4.2.

4.2. Coarse space with Neumann boundary conditions. In order to improve the coarse space and, in particular, to be able to prove an estimate for the condition number, we introduce some slight but significant modifications to the method. In particular, we define a new type of MsFEM basis functions by prescribing different values on the edges, we slightly modify the right-hand side of the eigenvalue problem, and we replace the left-hand side of the eigenvalue problem.

We first define $\eta_{i j}^{k h}$ to be a slab of $k$ layers of fine elements around the edge $e_{i j}$; cf. Figure 4.1. For the definition of the modified MsFEM basis functions, we replace the definition of the values on the edges (2.8) by the following: find $\hat{\varphi}_{P} \in V^{h}\left(e_{i j}\right)$ such that

$$
\begin{aligned}
a_{\eta_{i j}^{k h}}\left(\mathcal{H}_{e_{i j} \rightarrow \eta_{i j}^{k h}}\left(\hat{\varphi}_{P}\right), \mathcal{H}_{e_{i j} \rightarrow \eta_{i j}^{k h}}(\theta)\right) & =0 & & \forall \theta \in V_{0}^{h}\left(e_{i j}\right), \\
\hat{\varphi}_{P}\left(P^{\prime}\right) & =\delta_{P, P^{\prime}} & & \forall \text { vertices } P^{\prime} .
\end{aligned}
$$

As before, the interface values of $\hat{\varphi}_{P}$ are extended discrete harmonically into the interior according to (2.7). We denote the resulting MsFEM type coarse space as

$$
V_{\mathrm{MsFEM}-\hat{\mathrm{O}}, \mathrm{k}}:=\left(\bigoplus_{\mathfrak{v} \in \mathcal{V}} \operatorname{span}\left\{\hat{\varphi}_{P}\right\}\right) .
$$

If $\eta_{i j}^{k h}=\Omega_{e_{i j}}:=\Omega_{i} \cup \Omega_{j} \cup e_{i j}$, i.e., if the slab coincides with the complete subdomains, then we denote the space as $V_{\mathrm{MsFEM}-\mathrm{O}}$.

For the right-hand side of the eigenvalue problem, instead of using a scaled $L^{2}$-inner product on the edge, we use

$$
\tilde{b}_{e_{i j}}(u, v):=\frac{1}{h^{2}}\left(A w_{e_{i j}}(u), w_{e_{i j}}(v)\right)_{L^{2}\left(\Omega_{e_{i j}}\right)},
$$

where

$$
\begin{aligned}
w_{e_{i j}}: V_{0}^{h}\left(e_{i j}\right) & \rightarrow V_{0}^{h}\left(\Omega_{e_{i j}}\right), \\
v & \mapsto w_{e_{i j}}(v):= \begin{cases}v & \text { in all interior nodes of } e_{i j}, \\
0 & \text { on all other nodes in } \Omega_{e_{i j}}\end{cases}
\end{aligned}
$$


The corresponding norm is defined as

$$
\|u\|_{\tilde{b}, e_{i j}}^{2}:=\tilde{b}_{e_{i j}}(u, u) .
$$

In addition to that, we replace the Dirichlet boundary condition on the left-hand side of the eigenvalue problem by a Neumann boundary condition and obtain the eigenvalue problem:

$$
a_{\eta_{i j}^{k h}}\left(\mathcal{H}_{e_{i j} \rightarrow \eta_{i j}^{k h}}\left(\tau_{*, e_{i j}}\right), \mathcal{H}_{e_{i j} \rightarrow \eta_{i j}^{k h}}(\theta)\right)=\lambda_{*, e_{i j}} \tilde{b}_{e_{i j}}\left(\tau_{*, e_{i j}}, \theta\right) \quad \forall \theta \in V_{0}^{h}\left(e_{i j}\right) .
$$

In this version of the eigenvalue problem, no Dirichlet boundary conditions are prescribed on $\partial \eta_{i j}^{k h} \backslash e_{i j}$. The coarse basis functions $v_{*, e_{i j}}$ are again obtained by selecting eigenmodes with eigenvalues up to a certain threshold and by extending these values on the edge discrete harmonically to the subdomains, i.e., by solving (4.3).

The resulting coarse space depends on the width of $\eta_{i j}^{k h}$ and the threshold tol for the selection of the eigenvalues. If the width of $\eta_{i j}^{k h}$ consists of $k$ layers of fine elements in each of the subdomains $\Omega_{i}$ and $\Omega_{j}$, then the resulting coarse space is

$$
V_{\mathrm{ACMS}-\mathrm{N}, \mathrm{k}}^{\text {tol }}=V_{\mathrm{MsFEM}-\hat{\mathrm{O}}, \mathrm{k}} \oplus\left(\bigoplus_{\substack{e \subset \Gamma \\ e \text { edge }}} \operatorname{span}\left\{v_{k, e}: \lambda_{k, e} \leq t o l\right\}\right),
$$

and if $\eta_{i j}^{k h}=\Omega_{e_{i j}}$, then the coarse space is denoted as $V_{\mathrm{ACMS}-\mathrm{N}}^{t o l}$; see Section 6 for the proof of the condition number bound of the corresponding Schwarz operator.

4.3. Using a lumped mass matrix. The solution of generalized eigenvalue problems can be expensive, and therefore a typical criticism concerns the construction of the adaptive coarse spaces (in the off line stage). In order to reduce the computational cost for the solution of the generalized eigenvalue problem, one can lump the mass matrix on the right-hand side.

In particular, for the edge $e_{i j}$, both eigenvalue problems (4.2) and (4.8) can be written in discrete form as

$$
S_{e_{i j}} V=\lambda B_{e_{i j}} V
$$

using the Schur complement $S_{e_{i j}}$ where all degrees of freedom except for those on the interior edge have been eliminated. Depending on which version of the ACMS coarse space is used, zero Dirichlet boundary conditions have been applied only to endpoints of the edge (Neumann version) or to all degrees of freedom on $\left(\partial \Omega_{i} \cup \partial \Omega_{j}\right) \backslash e_{i j}$ (Dirichlet version). The matrix $B_{e_{i j}}$ corresponds to the scaled mass matrix on the edge, i.e., to the discretization of the bilinear form (4.5) (Neumann version) or (4.1) (Dirichlet version).

Now, since the mass matrix is spectrally equivalent to its diagonal, we can replace $B_{e_{i j}}$ by $\operatorname{diag}\left(B_{e_{i j}}\right)$ in (4.9) and obtain

$$
\operatorname{diag}\left(B_{e_{i j}}\right)^{-1} S_{e_{i j}} V=\lambda V
$$

or, in a symmetric version,

$$
\operatorname{diag}\left(B_{e_{i j}}\right)^{-1 / 2} S_{e_{i j}} \operatorname{diag}\left(B_{e_{i j}}\right)^{-1 / 2} W=\lambda W
$$

with $\operatorname{diag}\left(B_{e_{i j}}\right)^{1 / 2} V=W$ in a computationally inexpensive step.

Thus, we are able to solve a standard eigenvalue problem instead of a generalized eigenvalue problem. We denote the corresponding coarse spaces with a bar, i.e., as $V_{\mathrm{ACMS}-\overline{\mathrm{D}}}^{t o l}$, $V_{\text {ACMS- }}^{\text {tol }}$. Accordingly, we write $V_{\text {ACMS }-\overline{\mathrm{N}}, \mathrm{k}}^{\text {tol }}$ for the slab version.

As we will observe in Section 7, lumping the mass matrix does not affect the performance of the preconditioner. 
4.4. Properties of the spectral projection. We consider the projection onto the space spanned by the discrete harmonic extensions of the selected eigenfunctions, i.e., by $\left\{v_{k, e}\right\}_{\substack{e \subset \Gamma \\ e \text { edge }}}$,

$$
\Pi v:=\sum_{\substack{e \subset \Gamma \\ e \text { edge }}} \sum_{\lambda_{k, e} \leq t o l} \tilde{b}_{e}\left(v, v_{k, e}\right) v_{k, e} .
$$

This projection, which will be part of the construction of a stable decomposition of the twolevel Schwarz preconditioner in Section 6, has some typical properties which are summarized in the following lemma for the Neumann version of the eigenvalue problem; cf. (4.8). However, the properties in case of the Dirichlet version can be shown analogously. The proof of the next lemma is based on arguments from classical spectral theory and follows arguments which are standard in the theory of adaptive coarse spaces; see, e.g., [30, Lemma 5.3], [38, Lemma 2.11], and [31, Lemma 4.6].

LEMMA 4.1. For an edge e, consider the eigenvalue problem (4.8) and the corresponding eigenpairs $\left\{\left(\tau_{k, e}, \lambda_{k, e}\right)\right\}_{k=1}^{\operatorname{dim}\left(V_{0}^{h}(e)\right)}$, and let $v_{k, e}$ be the discrete harmonic extension of $\tau_{k, e}$; cf. (4.3). Then, the projection

$$
\Pi_{e} v:=\sum_{\lambda_{k, e} \leq t o l} \tilde{b}_{e}\left(v, v_{k, e}\right) v_{k, e}
$$

is orthogonal with respect to the bilinear form

$$
a_{*, e, \eta}(u, v):=a_{\eta}\left(\mathcal{H}_{e \rightarrow \eta}(u), \mathcal{H}_{e \rightarrow \eta}(v)\right),
$$

and therefore

$$
|v|_{a, *, e, \eta}^{2}=\left|\Pi_{e} v\right|_{a, *, e, \eta}^{2}+\left|v-\Pi_{e} v\right|_{a, *, e, \eta}^{2} .
$$

The semi-norm is defined as

$$
|u|_{a, *, e, \eta}^{2}:=a_{*, e, \eta}(u, u)
$$

In addition, the estimate

$$
\left\|v-\Pi_{e} v\right\|_{\tilde{b}, e}^{2} \leq \frac{1}{t o l}\left|v-\Pi_{e} v\right|_{a, *, e, \eta}^{2}
$$

holds.

Proof. We have

$$
a_{*, e, \eta}\left(v_{k, e}, v_{l, e}\right)=a_{*, e, \eta}\left(\tau_{k, e}, \tau_{l, e}\right)=\tilde{b}_{e}\left(\tau_{k, e}, \tau_{l, e}\right)=\tilde{b}_{e}\left(v_{k, e}, v_{l, e}\right)=0 \quad \forall k \neq l
$$

and

$$
\begin{gathered}
\left|v_{k, e}\right|_{a, *, e, \eta}^{2}=\left|\tau_{k, e}\right|_{a, *, e, \eta}^{2}=\lambda_{k, e} \\
\left\|v_{k, e}\right\|_{\tilde{b}, e}^{2}=\left\|\tau_{k, e}\right\|_{\tilde{b}, e}^{2}=1
\end{gathered}
$$

Therefore, since

$$
v=\sum_{k=1}^{\operatorname{dim}\left(V_{0}^{h}(e)\right)} \tilde{b}_{e}\left(v, v_{k, e}\right) v_{k, e} \quad \forall v \in V_{0}^{h}(e)
$$



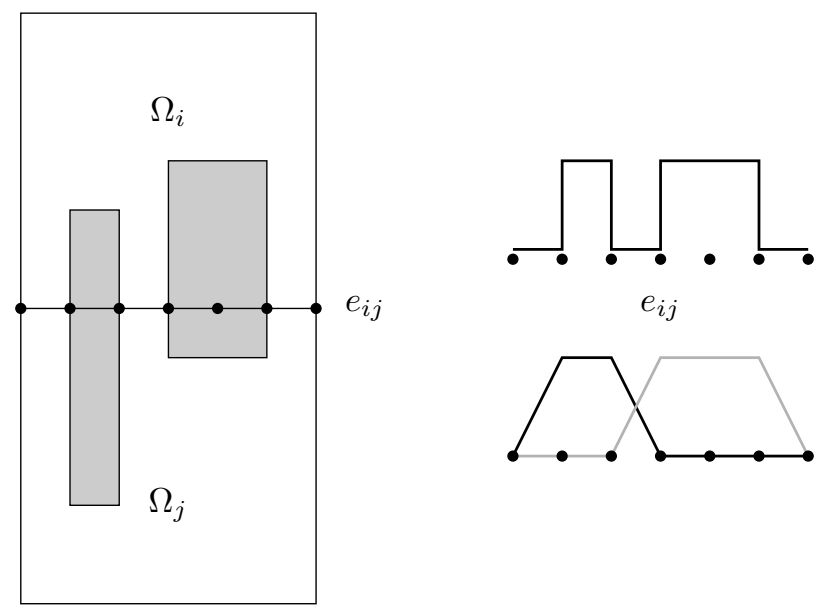

FIG. 4.2. Heuristic construction of the edge-based coarse basis functions in ACMS-R in order to avoid eigenvalue problems; see Section 4.5: coefficient function on the two subdomains $\Omega_{i}$ and $\Omega_{j}$ (left), coefficient function on the edge $e_{i j}$ (top,right), and the resulting two coarse basis functions (bottom,right).

we obtain

$$
\begin{aligned}
|v|_{a, *, e, \eta}^{2} & =\left|\sum_{\lambda_{k, e} \leq t o l} \tilde{b}_{e}\left(v, v_{k, e}\right) v_{k, e}\right|_{a, *, e, \eta}^{2}+\left|\sum_{\lambda_{k, e}>t o l} \tilde{b}_{e}\left(v, v_{k, e}\right) v_{k, e}\right|_{a, *, e, \eta}^{2} \\
& =\left|\Pi_{e} v\right|_{a, *, e, \eta}^{2}+\left|v-\Pi_{e} v\right|_{a, *, e, \eta}^{2} .
\end{aligned}
$$

Moreover,

$$
\begin{aligned}
\left\|v-\Pi_{e} v\right\|_{\tilde{b}, e}^{2} & =\left\|\sum_{\lambda_{k, e}>t o l} \tilde{b}_{e}\left(v, v_{k, e}\right) v_{k, e}\right\|_{\tilde{b}, e}^{2}=\sum_{\lambda_{k, e}>t o l} \tilde{b}_{e}\left(v, v_{k, e}\right)\left\|v_{k, e}\right\|_{\tilde{b}, e}^{2} \\
& =\sum_{\lambda_{k, e}>t o l} \tilde{b}_{e}\left(v, v_{k, e}\right) \frac{1}{\lambda_{k, e}}\left|v_{k, e}\right|_{a, *, e, \eta}^{2} \leq \frac{1}{t o l} \sum_{\lambda_{k, e}>t o l} \tilde{b}_{e}\left(v, v_{k, e}\right)\left|v_{k, e}\right|_{a, *, e, \eta}^{2} \\
& =\frac{1}{t o l}\left|\sum_{\lambda_{k, e}>t o l} \tilde{b}_{e}\left(v, v_{k, e}\right) v_{k, e}\right|_{a, *, e, \eta}^{2}=\frac{1}{t o l}\left|v-\Pi_{e} v\right|_{a, *, e, \eta}^{2} \cdot
\end{aligned}
$$

Since

$$
\Pi v=\sum_{e \subset \Gamma} \Pi_{e} v,
$$

the projection $\Pi$ inherits the bounds for the local projections $\Pi_{e}$. In particular, this will be essential in the proofs of Section 6.

4.5. Heuristic construction of edge functions. In order to reduce the cost for the computation of the edge-based coupling basis functions even further, one can construct the trace values by inspecting the values of the coefficient function. In particular, we construct one basis function for each heterogeneity with a high coefficient intersecting an edge and extend these 


\section{ETNA}

Kent State University and

Johann Radon Institute (RICAM)

values discrete harmonically to the interior of the subdomain. Precisely, we set the functions to one for those degrees of freedom which belong to an element inside a heterogeneity with a high coefficient and to zero elsewhere; cf. Figure 4.2. Therefore, we define a tolerance tol which characterizes the threshold for the identification of a coefficient jump: we identify a coefficient jump if the contrast of the coefficient, $\frac{A_{t_{1}}}{A_{t_{2}}}$ or $\frac{A_{t_{2}}}{A_{t_{1}}}$, of two neighboring elements $t_{1}$ and $t_{2}$ is larger than tol.

We denote the resulting coarse space, which consists of the constructed edge-based basis functions and the ACMS vertex-specific basis functions, as $V_{\mathrm{ACMS}-\mathrm{R}}^{\text {tol }}$. Let us note that the vectors constructed from this approach could also be used in other ACMS approaches as initial values in iterative methods for eigenvalue problems. The first results for the use of the $V_{\mathrm{ACMS}-\mathrm{R}}^{\text {tol }}$ coarse space can be found in [21] and in the master thesis of the third author.

5. Related adaptive coarse spaces for overlapping Schwarz. While there are several different approaches to define adaptive coarse spaces for overlapping Schwarz preconditioners, we would like to mention two of them (in Section 5.2 and Section 5.3) which are closely related to our approach. But first, we will describe an early approach by Galvis and Efendiev [16] in the subsequent Section 5.1.

5.1. Local spectral multiscale coarse spaces. In [16], Galvis and Efendiev proposed to circumvent the need for a weighted Poincare inequality

$$
\int_{\Omega} A(x) v^{2} d x \leq C \int_{\Omega} A(x)(\nabla v)^{2} d x
$$

with a constant $C$ which depends on the contrast of the coefficient $\frac{\max _{x \in \Omega} A(x)}{\min _{x \in \Omega} A(x)}$ by using eigenfunctions as coarse space functions.

Therefore, the eigenvalue problem

$$
\operatorname{div}\left(A(x) \nabla \psi_{k}^{\omega_{P}}\right)=\lambda_{i} A(x) \psi_{k}^{\omega_{P}}
$$

on the union of the neighboring subdomains ("coarse grid blocks")

$$
\omega_{P}:=\bigcup_{T \in \tau_{H}, P \in \bar{T}} T
$$

of the vertex $P$ of the coarse mesh is considered. The discrete form of the eigenvalue problem is: find $\psi_{i}^{\omega_{P}} \in \tilde{V}^{h}\left(\omega_{P}\right):=\left\{v \in V^{h}\left(\omega_{P}\right):\left.v\right|_{\partial \omega_{P} \cap \partial \Omega}=0\right\}$ such that

$$
A^{\omega_{P}} \psi_{k}^{\omega_{P}}=\lambda_{k} M^{\omega_{P}} \psi_{k}^{\omega_{P}},
$$

where $A^{\omega_{P}}$ is the stiffness matrix obtained from the bilinear form $a_{\omega_{P}}(\cdot, \cdot)$ and $M^{\omega_{P}}$ is the generalized mass matrix obtained from the bilinear form

$$
\int_{\omega_{P}} A(x) u v d x
$$

Now let $\left\{\chi_{P}\right\}_{P} \underset{P \in \Gamma}{P \in{ }_{\text {vertex }}}$ be a partition of unity, which is subordinated to the covering $\left\{\omega_{P}\right\}_{P \text { vertex }}^{P \in \Gamma}$ of $\Omega$ with $\chi_{P} \in V^{h}(\Omega)$ and $\left|\nabla \chi_{P}\right| \leq \frac{1}{H}$. Then, the coarse basis functions are given as

$$
\Phi_{P, k}=I^{h}\left(\chi_{P} \psi_{k}^{\omega_{P}}\right) \quad \text { for } P \in \Gamma \text { vertex and } 1 \leq i \leq L_{P},
$$




\section{ETNA}

Kent State University and

Johann Radon Institute (RICAM)

where $L_{P}$ is the number of eigenfunctions selected from the eigenvalue problem on the patch $\omega_{P}$. The local spectral multiscale coarse space is then defined as

$$
V_{L S M}:=\left\{\Phi_{P, k}: P \in \Gamma, 1 \leq k \leq L_{P}\right\} .
$$

For a two-level Schwarz preconditioner using this coarse space, the authors prove the condition number bound

$$
\kappa\left(M_{L S M}^{-1} K\right) \leq C\left(1+\frac{H^{2}}{\delta^{2}}\right),
$$

where the constant $C$ depends on the number of eigenfunctions selected for the coarse space but not on the contrast of the coefficient function and the mesh size. However, the solution of the eigenvalue problems (5.1) is quite expensive: each eigenvalue problem is defined on the (coarse) neighborhood $\omega_{P}$ of a coarse node, i.e., it involves all neighboring subdomains of a vertex in the domain decomposition. Moreover, in this approach, unnecessary coarse space functions can be added to the coarse space resulting from the interior part of the subdomain.

As a result, improved approaches were proposed later. For example in [17], the authors have presented an improved version, where the dimension of the coarse space is reduced by introducing a suitable second partition of unity which modifies the scaling of the mass matrix. Hence, basis functions which correspond to interior inclusions are eliminated.

We will now describe the two approaches, [11] and [19], which are more closely related to our new algorithm. The authors there, like us, use smaller eigenvalue problems, i.e., eigenvalue problems on lower-dimensional manifolds instead of volumes in order to further reduce computational costs.

5.2. Coarse spaces based on local Dirichlet-to-Neumann maps. In [11], Dolean et al. have introduced a coarse space that is related to the ACMS approach. However, the corresponding eigenvalue problems are defined on the complete boundary of the overlapping subdomains, i.e., they are defined using the Schur complements on the subdomain boundaries $\partial \Omega_{i}^{\prime}$; see (5.2).

In particular, on each overlapping subdomain $\Omega_{i}^{\prime}$, the eigenvalue problem is given by

$$
\begin{aligned}
\operatorname{div}\left(A(x) \nabla v_{k}\right) & =0 & & \text { in } \Omega_{i}^{\prime}, \\
A(x) \frac{\partial v_{k}}{\partial n_{i}} & =\lambda_{k} A(x) v_{k} & & \text { on } \partial \Omega_{i}^{\prime},
\end{aligned}
$$

where $\frac{\partial}{\partial n_{i}}$ is the derivative in normal direction. The corresponding variational formulation is

$$
\int_{\Omega_{i}^{\prime}} A(x) \nabla v_{k} \cdot \nabla w d x=\lambda_{k} \int_{\partial \Omega_{i}^{\prime}} \operatorname{tr}_{i}(A(x)) v_{k} w d s \quad \forall w \in H^{1}\left(\Omega_{i}^{\prime}\right),
$$

where $\operatorname{tr}_{i} A(x):=\lim _{y \in \Omega_{i}^{\prime} \rightarrow x} A(x)$ for a.e. $x \in \partial \Omega_{i}^{\prime}$. In discrete form, the eigenvalue problem can be written as

$$
S_{\Gamma^{\prime}}^{(i)} v_{k, \Gamma^{\prime}}^{(i)}=\lambda_{k} M_{\Gamma^{\prime}}^{(i)} v_{k, \Gamma^{\prime}}^{(i)}
$$

Here, $M_{\Gamma^{\prime}}^{(i)}$ corresponds to the $1 \mathrm{D}$ mass matrix on $\partial \Omega_{i}^{\prime}$, and $S_{\Gamma^{\prime}}^{(i)}$ corresponds to the Schur complement where the interior degrees of freedom of the overlapping subdomains have been 
eliminated. Then, $v_{k, \Gamma}^{(i)}$ is extended discrete harmonically to $\Omega_{i}^{\prime}$ and denoted by $v_{k}^{(i)}$. The corresponding coarse space is given by

$$
V_{\mathrm{DtN}}:=\operatorname{span}\left\{\Phi_{i, k}^{H}: 1 \leq i \leq N \text { and } 1 \leq k \leq m_{i}\right\},
$$

where $m_{i}$ is the number of eigenfunctions selected in the subdomain $\Omega_{i}^{\prime}$. The basis functions are defined by

$$
\Phi_{i, k}^{H}:=I^{h}\left(\chi_{i} v_{k}^{(i)}\right)
$$

and the functions $\left\{\chi_{i}\right\}_{i=1}^{N}$ form a partition of unity corresponding to the overlapping decomposition $\left\{\Omega_{i}^{\prime}\right\}_{i=1}^{N}$ with

$$
\chi_{i}\left(x_{j}\right):=\frac{\operatorname{dist}\left(x_{j}, \partial \Omega_{i}^{\prime}\right)}{\sum_{x_{j} \in \Omega_{l}^{\prime}} \operatorname{dist}\left(x_{j}, \partial \Omega_{l}^{\prime}\right)} .
$$

In order to prove the condition number estimate

$$
\kappa\left(M_{\mathrm{DtN}}^{-1} K\right) \leq C+\max _{i=1}^{N} \frac{1}{\delta \lambda_{m_{i}+1}^{(i)}},
$$

the authors assume a quasi-monotone coefficient function $A$. Here, $\delta$ denotes the width of the overlap.

Note that the eigenvalue problems are still rather large since each corresponds to the degrees of freedom on the complete boundary of a single overlapping subdomain.

5.3. Spectral harmonically enriched multiscale coarse space. In $[18,19]$, Gander et al. have introduced a coarse space that is very closely related to our approach of using the ACMS space as a coarse space: first, MsFEM basis functions corresponding to the vertices of the decomposition are used in the coarse space, second, eigenvalue problems on the degrees of freedom of the edges of the nonoverlapping decomposition are employed.

In particular, the eigenvalue problem

$$
\bar{a}_{e_{i j}}\left(\psi_{e_{i j}}^{k}, v\right)=\lambda_{e_{i j}}^{k} \bar{b}_{e_{i j}}\left(\psi_{e_{i j}}^{k}, v\right) \quad \forall v \in V_{0}^{h}\left(e_{i j}\right)
$$

is considered, where

$$
\begin{aligned}
\bar{a}_{e_{i j}}(u, v) & :=\left(A_{e_{i j}, \max }(x) D_{x^{t}} u, D_{x^{t}} v\right), \\
\bar{b}_{e_{i j}}(u, v) & :=h^{-1} \sum_{x_{k} \in e_{i j}} \beta_{k} u\left(x_{k}\right) v\left(x_{k}\right) \quad \text { and } \\
\beta_{k} & :=\sum_{\left\{t \in \tau_{h}: k \in \operatorname{dof}(t)\right\}} A_{t} .
\end{aligned}
$$

Here, $A_{e_{i j} \text {, max }}$ is defined as in (2.9), $A_{t}$ is the constant coefficient on the element $t \in \tau_{h}, D_{x^{t}}$ denotes the tangent derivative with respect to the edge $e_{i j}$, and $x_{k}$ correspond to the finite element nodes on the edge. Eigenfunctions corresponding to the first $m_{e_{i j}}$ eigenvalues are selected and extended discrete harmonically by solving

$$
\begin{array}{rlrl}
a_{\Omega_{l}}\left(p_{e_{i j}}^{k}, v\right) & =0 & & \forall v \in V_{0}^{h}\left(\Omega_{l}\right), l=1, \ldots, N, \\
p_{e_{i j}}^{k}=\psi_{e_{i j}}^{k} & & \text { on } e_{i j}, \\
p_{e_{i j}}^{k} & =0 & & \text { on } \Gamma \backslash e_{i j} .
\end{array}
$$


The Spectral Harmonically Enriched Multiscale (SHEM) coarse space is then given by

$$
V_{\text {SHEM }}:=V_{\mathrm{MsFEM}-\mathrm{O}} \oplus\left(\bigoplus_{e_{i j} \subset \Gamma} \operatorname{span}\left\{p_{e_{i j}}^{k}: k \leq m_{e_{i j}}\right\}\right) .
$$

Using this choice, the authors are able to prove an estimate for the condition number of the preconditioned system

$$
\kappa\left(M_{\mathrm{SHEM}}^{-1} A\right) \leq C\left(1+\frac{1}{\lambda_{m+1}}\right),
$$

where $\lambda_{m+1}:=\max _{e_{i j} \subset \Gamma}\left\{\lambda_{e_{i j}}^{m_{e_{i j}}}\right\}$.

The authors thus use the bilinear form on the edge (rather than a Schur complement). The eigenvalue problems are small and local and therefore inexpensive to set up and solve. However, a drawback of this coarse space is that, due to the reduction of the eigenvalue problem to the edges, the coarse space can become large for certain coefficient functions where a much smaller coarse space would be sufficient; cf. Section 7, where we observe that this smaller coarse space will be found by the non-economic versions of the new methods described in this paper, i.e., by using the eigenvalue problems from Section 4.1 or Section 4.2. Of course, if in the economic versions the slab is wide enough, then the resulting adaptive method will also be successful. Note that among our methods, only the methods from Section 4.2 are covered by our theory in Section 6. The SHEM coarse space could be interpreted as the limit case of our slab variants, where the slab is reduced such that no extensions are used. Since we always use extensions in our eigenvalue problems, in our theory, we never have to estimate from volumes to edges and vice versa.

6. Convergence analysis for the overlapping Schwarz method with the ACMS coarse space. To prove an estimate for the convergence of the OS-ACMS preconditioner, we essentially have to prove the existence of a stable decomposition; cf., e.g., [40]. Therefore, we have to provide a suitable coarse interpolation $I_{0}$ into the coarse space

$$
V_{0}=\left(\bigoplus_{\substack{P \in \Gamma \\ P \text { vertex }}} \operatorname{span}\left\{\hat{\varphi}_{P}\right\}\right) \oplus\left(\bigoplus_{\substack{e \subset \Gamma \\ e \text { edge }}} \operatorname{span}\left\{v_{k, e}: \lambda_{k, e} \leq t o l\right\}\right),
$$

in particular, into the spaces $V_{A C M S-N}^{t o l}$ or $V_{A C M S-N, k}^{t o l}$. We construct $I_{0}$ by pointwise interpolation

$$
I_{\operatorname{MsFEM}} u:=\sum_{\substack{P \in \Gamma \\ P \text { vertex }}} u(P) \hat{\varphi}_{P}
$$

to the coarse multiscale space $V_{\mathrm{MsFEM}-\mathrm{O}, \mathrm{k}}$ (see also (4.4)) and the projection $\Pi$ onto the space spanned by the edge-based coupling eigenfunctions; cf. (4.10).

We start with the proof of a lemma which states the $a_{*, e, \eta}$-stability of the MsFEM interpolation operator.

LEMMA 6.1. The MSFEM interpolation operator, which is defined in (6.1), is stable with

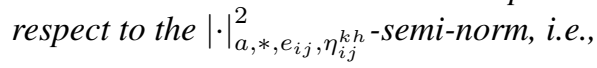

$$
\left|I_{\mathrm{MsFEM}} v\right|_{a, *, e_{i j}, \eta_{i j}^{k h}}^{2} \leq|v|_{a, *, e_{i j}, \eta_{i j}^{k h}}^{2} \quad \forall v \in V^{h}\left(e_{i j}\right) .
$$


Proof. The MsFEM interpolation is exact on the vertices of the nonoverlapping decomposition, i.e.,

$$
I_{\operatorname{MsFEM}} v(P)=v(P)
$$

for all vertices $P \in \Gamma$. Furthermore, the MsFEM basis functions are defined as energy minimizing functions with respect to $|\cdot|_{a, *, e_{i j}, \eta_{i j}^{k}}$ and the corresponding boundary values; cf. (4.4). Therefore, $I_{\mathrm{MsFEM}} v$ is the energy minimal extension of the values of $v$ on the vertices to the edges, and thus

$$
\left|I_{\mathrm{MsFEM}} v\right|_{a, *, e_{i j}, \eta_{i j}^{k h}}^{2} \leq|v|_{a, *, e_{i j}, \eta_{i j}^{k h}}^{2} \quad \forall v \in V^{h}\left(e_{i j}\right) .
$$

We define the coarse component of the stable decomposition as

$$
u_{0}:=I_{0} u:=I_{\mathrm{MsFEM}} u+\Pi\left(u-I_{\mathrm{MsFEM}} u\right) \in V_{0},
$$

which we use to prove the existence of a stable decomposition. For the proof, we also need the following useful lemma.

LEMMA 6.2. Let $u \in V^{h}(\Omega), u_{0}:=I_{0} u:=I_{\operatorname{MsFEM}} u+\Pi\left(u-I_{\operatorname{MsFEM}} u\right)$. Then,

$$
\left|w_{e_{i j}}\left(u-u_{0}\right)\right|_{a, \Omega_{i}}^{2} \leq \frac{4 C_{\mathrm{inv}}}{\text { tol }}\left(|u|_{a, \Omega_{i}}^{2}+|u|_{a, \Omega_{j}}^{2}\right),
$$

where $C_{\mathrm{inv}}>0$ is a constant arising from the use of an inverse equality on the elements. It is independent of $H, h$, and the contrast of the coefficient function. The operator $w_{e_{i j}}$ is defined in (4.6).

REMARK 6.3. The constant $C_{\text {inv }}$ depends only on the shape parameter of the triangulation and the polynomial degree of the shape functions; see, e.g., [41, Section 3.6], where also an explicit upper bound for $C_{\text {inv }}$ is given.

Proof. From the construction of $w_{e_{i j}}$ and a standard inverse inequality, we obtain

$$
\left|w_{e_{i j}}\left(u-u_{0}\right)\right|_{a, \Omega_{i}}^{2} \leq C_{\mathrm{inv}}\left\|u-u_{0}\right\|_{\tilde{b}, e_{i j}}^{2} .
$$

Using the properties of the projection $\Pi$, cf. Lemma 4.1, we obtain

$$
\begin{aligned}
\left\|u-u_{0}\right\|_{\tilde{b}, e_{i j}}^{2} & =\left\|u-I_{\operatorname{MsFEM}} u-\Pi\left(u-I_{\operatorname{MsFEM}} u\right)\right\|_{\tilde{b}, e_{i j}}^{2} \\
& \leq \frac{1}{t o l}\left|u-I_{\operatorname{MsFEM}} u-\Pi\left(u-I_{\operatorname{MsFEM}} u\right)\right|_{a, *, e_{i j}, \eta_{i j}^{k h}}^{2} \\
& \leq \frac{1}{t o l}\left|u-I_{\operatorname{MsFEM}} u\right|_{a, *, e_{i j}, \eta_{i j}^{k h}}^{2} .
\end{aligned}
$$

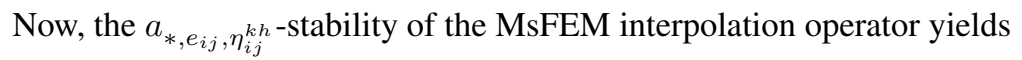

$$
\begin{aligned}
\left|u-I_{\operatorname{MsFEM}} u\right|_{a, *, e_{i j}, \eta_{i j}^{k h}}^{2} & \leq 2|u|_{a, *, e_{i j}, \eta_{i j}^{k h}}^{2}+2\left|I_{\mathrm{MsFEM}} u\right|_{a, *, e_{i j}, \eta_{i j}^{k h}}^{2} \\
& \leq 4|u|_{a, *, e_{i j}, \eta_{i j}^{k h}}^{2} \\
& =4\left|\mathcal{H}_{e_{i j} \rightarrow \eta_{i j}^{k h}}(u)\right|_{a, \eta_{i j}^{k h}}^{2} \\
& \leq 4\left(|u|_{a, \Omega_{i}}^{2}+|u|_{a, \Omega_{j}}^{2}\right)
\end{aligned}
$$

cf. Lemma 6.1. We would like to point out that the harmonic extension in (6.2) is essential to treat the comb-like coefficients as in Figure 7.3. If the slab $\eta_{i j}^{k h}$ does not cover the whole 


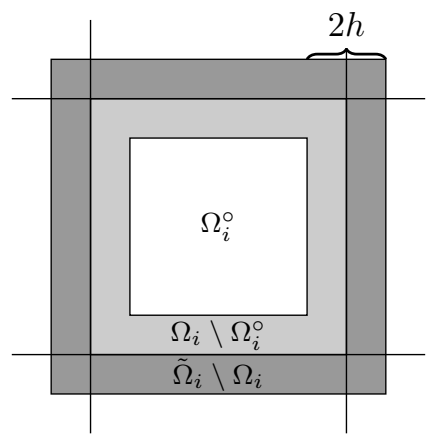

FIG. 6.1. In our proof of theorem 6.4, we consider a partition of unity corresponding to an overlapping decomposition with overlap $h$. The corresponding regions $\Omega_{i}^{\circ}$ (white), $\Omega_{i} \backslash \Omega_{i}^{\circ}$ (light gray), and $\tilde{\Omega}_{i} \backslash \Omega_{i}$ (dark gray) are depicted.

high-coefficient structure, then the resulting coarse space may grow significantly; cf. Table 7.3. This is also the case if the technique in [19, Equation 23] is used. This highlights the fact that the SHEM coarse space can be viewed as the limit case where the width of the slab is zero.

Finally, we obtain

$$
\left|w_{e_{i j}}\left(u-u_{0}\right)\right|_{a, \Omega_{i}}^{2} \leq \frac{4 C_{\mathrm{inv}}}{t o l}\left(|u|_{a, \Omega_{i}}^{2}+|u|_{a, \Omega_{j}}^{2}\right)
$$

Now, we are able to prove the existence of a stable decomposition.

LEMma 6.4 (Stable Decomposition). For each $v \in V=V^{h}(\Omega)$, there exists a decomposition $u=\sum_{i=0}^{N} R_{i}^{T} u_{i}$ with $u_{i} \in V_{i}=V^{h}\left(\Omega_{i}^{\prime}\right)$ such that

$$
\sum_{i=0}^{N}\left|u_{i}\right|_{a, \Omega_{i}^{\prime}}^{2} \leq C_{0}^{2}|u|_{a, \Omega}^{2}
$$

where $C_{0}^{2}=\left(20+\frac{118\left(N^{e}\right)^{2} C_{\mathrm{inv}}}{t o l}\right)$. The constant $N^{e}$ denotes the maximum number of edges that a single subdomain may have.

Proof. We first consider the estimate of the coarse component and proceed subdomain by subdomain. Using

$$
\left|u_{0}\right|_{a, \Omega}^{2}=\sum_{i=1}^{N}\left|u_{0}\right|_{a, \Omega_{i}}^{2}
$$

Lemma 6.2, and the fact that $u_{0}$ is discrete harmonic on each subdomain $\Omega_{i}$, we obtain

$$
\begin{aligned}
\left|u_{0}\right|_{a, \Omega_{i}}^{2} & \leq 2\left(\left|\mathcal{H}_{\partial \Omega_{i} \rightarrow \Omega_{i}}(u)\right|_{a, \Omega_{i}}^{2}+\left|\mathcal{H}_{\partial \Omega_{i} \rightarrow \Omega_{i}}\left(u-u_{0}\right)\right|_{a, \Omega_{i}}^{2}\right) \\
& \leq 2\left(|u|_{a, \Omega_{i}}^{2}+N^{e} \sum_{e_{i j} \subset \partial \Omega_{i}}\left|w_{e_{i j}}\left(u-u_{0}\right)\right|_{a, \Omega_{i}}^{2}\right) \\
& \leq 2|u|_{a, \Omega_{i}}^{2}+\frac{8 N^{e} C_{\mathrm{inv}}}{t o l} \sum_{e_{i j} \subset \partial \Omega_{i}}\left(|u|_{a, \Omega_{i}}^{2}+|u|_{a, \Omega_{j}}^{2}\right) .
\end{aligned}
$$


Now, we consider the local components $u_{i}:=I^{h}\left(\theta_{i}\left(u-u_{0}\right)\right)$ based on the partition of unity $\left\{\theta_{i}\right\}_{i=1}^{N}$ with

$$
\theta_{i}\left(x_{j}\right):=\frac{\operatorname{dist}\left(x_{j}, \partial \tilde{\Omega}_{i}\right)}{\sum_{x_{j} \in \tilde{\Omega}_{k}} \operatorname{dist}\left(x_{j}, \partial \tilde{\Omega}_{k}\right)}
$$

and $\left\{\tilde{\Omega}_{i}\right\}_{i=1}^{N}$ being an overlapping decomposition with overlap $h$ corresponding to the nonoverlapping decomposition $\left\{\Omega_{i}\right\}_{i=1}^{N}$; see, e.g., [40, Lemma 3.4]. Note that this overlapping decomposition is only used for this proof and can therefore differ from the overlapping decomposition $\left\{\Omega_{i}^{\prime}\right\}_{i=1}^{N}$ used in the first level of the preconditioner; cf. Section 3. However, since the overlap of the decomposition $\left\{\Omega_{i}^{\prime}\right\}_{i=1}^{N}$ is at least $h$, we always have $\tilde{\Omega}_{i} \subseteq \Omega_{i}^{\prime}$, and thus $u_{i} \in V_{i}$.

Therefore,

$$
u=\sum_{i=0}^{N} R_{i}^{T} u_{i}
$$

and

$$
\left|u_{i}\right|_{a, \Omega_{i}^{\prime}}^{2}=\left|u_{i}\right|_{a, \tilde{\Omega}_{i}}^{2}=\left|u_{i}\right|_{a, \tilde{\Omega}_{i} \backslash \Omega_{i}}^{2}+\left|u_{i}\right|_{a, \Omega_{i} \backslash \Omega_{i}^{\circ}}^{2}+\left|u_{i}\right|_{a, \Omega_{i}^{\circ}}^{2} ;
$$

cf. Figure 6.1 for a graphical representation of the regions $\tilde{\Omega}_{i} \backslash \Omega_{i}, \Omega_{i} \backslash \Omega_{i}^{\circ}$, and $\Omega_{i}^{\circ}$.

Now, analogously to (6.3), we have

$$
\begin{aligned}
\left|u_{i}\right|_{a, \Omega_{i}^{\circ}}^{2} & \leq\left|u-u_{0}\right|_{a, \Omega_{i}}^{2} \leq 2|u|_{a, \Omega_{i}}^{2}+2\left|u_{0}\right|_{a, \Omega_{i}}^{2} \\
& \leq 2|u|_{a, \Omega_{i}}^{2}+2\left(2|u|_{a, \Omega_{i}}^{2}+\frac{8 N^{e} C_{\mathrm{inv}}}{t o l} \sum_{e_{i j} \subset \partial \Omega_{i}}\left(|u|_{a, \Omega_{i}}^{2}+|u|_{a, \Omega_{j}}^{2}\right)\right) \\
& \leq 6|u|_{a, \Omega_{i}}^{2}+\frac{16 N^{e} C_{\mathrm{inv}}}{t o l} \sum_{e_{i j} \subset \partial \Omega_{i}}\left(|u|_{a, \Omega_{i}}^{2}+|u|_{a, \Omega_{j}}^{2}\right) .
\end{aligned}
$$

Furthermore, we have

$$
\begin{aligned}
\left|u_{i}\right|_{a, \Omega_{i} \backslash \Omega_{i}^{\circ}}^{2} & \leq 2\left|u_{i}-\left(u-u_{0}\right)\right|_{a, \Omega_{i} \backslash \Omega_{i}^{\circ}}^{2}+2\left|u-u_{0}\right|_{a, \Omega_{i} \backslash \Omega_{i}^{\circ}}^{2} \\
& \leq 2\left|I^{h}\left(\left(1-\theta_{i}\right)\left(u-u_{0}\right)\right)\right|_{a, \Omega_{i} \backslash \Omega_{i}^{\circ}}^{2}+4|u|_{a, \Omega_{i}}^{2}+4\left|u_{0}\right|_{a, \Omega_{i}}^{2} \\
& =2\left|\sum_{e_{i j} \subset \partial \Omega_{i}} I^{h}\left(\left(1-\theta_{i}\right) w_{e_{i j}}\left(u-u_{0}\right)\right)\right|_{a, \Omega_{i} \backslash \Omega_{i}^{\circ}}^{2}+4|u|_{a, \Omega_{i}}^{2}+4\left|u_{0}\right|_{a, \Omega_{i}}^{2} \\
& =2\left|\sum_{e_{i j} \subset \partial \Omega_{i}} \frac{1}{2} w_{e_{i j}}\left(u-u_{0}\right)\right|_{a, \Omega_{i} \backslash \Omega_{i}^{\circ}}^{2}+4|u|_{a, \Omega_{i}}^{2}+4\left|u_{0}\right|_{a, \Omega_{i}}^{2} \\
& \leq \frac{1}{2} N^{e} \sum_{e_{i j} \subset \partial \Omega_{i}}\left|w_{e_{i j}}\left(u-u_{0}\right)\right|_{a, \Omega_{i} \backslash \Omega_{i}^{\circ}}^{2}+4|u|_{a, \Omega_{i}}^{2}+4\left|u_{0}\right|_{a, \Omega_{i}}^{2}
\end{aligned}
$$




$$
\begin{aligned}
\leq & \frac{1}{2} N^{e} \sum_{e_{i j} \subset \partial \Omega_{i}}\left|w_{e_{i j}}\left(u-u_{0}\right)\right|_{a, \Omega_{i}}^{2}+4|u|_{a, \Omega_{i}}^{2}+\left.4|u|_{0}\right|_{a, \Omega_{i}} ^{2} \\
\leq & \frac{2 N^{e} C_{\mathrm{inv}}}{t o l} \sum_{e_{i j} \subset \partial \Omega_{i}}\left(|u|_{a, \Omega_{i}}^{2}+|u|_{a, \Omega_{j}}^{2}\right) \\
& +4|u|_{a, \Omega_{i}}^{2}+4\left(2|u|_{a, \Omega_{i}}^{2}+\frac{8 N^{e} C_{\mathrm{inv}}}{t o l} \sum_{e_{i j} \subset \partial \Omega_{i}}\left(|u|_{a, \Omega_{i}}^{2}+|u|_{a, \Omega_{j}}^{2}\right)\right) \\
\leq & 12|u|_{a, \Omega_{i}}^{2}+\frac{34 N^{e} C_{\mathrm{inv}}}{t o l} \sum_{e_{i j} \subset \partial \Omega_{i}}\left(|u|_{a, \Omega_{i}}^{2}+|u|_{a, \Omega_{j}}^{2}\right)
\end{aligned}
$$

and

$$
\begin{aligned}
\left|u_{i}\right|_{a, \tilde{\Omega}_{i} \backslash \Omega_{i}}^{2} & =\left|I^{h}\left(\theta_{i}\left(u-u_{0}\right)\right)\right|_{a, \tilde{\Omega}_{i} \backslash \Omega_{i}}^{2} \\
& \leq N^{e} \sum_{e_{i j} \subset \partial \Omega_{i}}\left|I^{h}\left(\theta_{i} w_{e_{i j}}\left(u-u_{0}\right)\right)\right|_{a, \tilde{\Omega}_{i} \backslash \Omega_{i}}^{2} \\
& \leq \frac{1}{4} N^{e} \sum_{e_{i j} \subset \partial \Omega_{i}}\left|w_{e_{i j}}\left(u-u_{0}\right)\right|_{a, \Omega_{j}}^{2} \\
& \leq \frac{N^{e} C_{\mathrm{inv}}}{t o l} \sum_{e_{i j} \subset \partial \Omega_{i}}\left(|u|_{a, \Omega_{i}}^{2}+|u|_{a, \Omega_{j}}^{2}\right) .
\end{aligned}
$$

In both derivations, we have used that $w_{e_{i j}}\left(u-u_{0}\right)=u-u_{0}$ on the closed edge $\bar{e}_{i j}$. Therefore, we get

$$
\begin{aligned}
\sum_{i=0}^{N}\left|u_{i}\right|_{a, \Omega}^{2} & =\sum_{i=1}^{N}\left(\left|u_{0}\right|_{a, \Omega_{i}}^{2}+\left|u_{i}\right|_{a, \tilde{\Omega}_{i} \backslash \Omega_{i}}^{2}+\left|u_{i}\right|_{a, \Omega_{i} \backslash \Omega_{i}^{\circ}}^{2}+\left|u_{i}\right|_{a, \Omega_{i}^{\circ}}^{2}\right) \\
& \leq \sum_{i=1}^{N}\left(20|u|_{a, \Omega_{i}}^{2}+\frac{59 N^{e} C_{\mathrm{inv}}}{t o l} \sum_{e_{i j} \subset \partial \Omega_{i}}\left(|u|_{a, \Omega_{i}}^{2}+|u|_{a, \Omega_{j}}^{2}\right)\right) \\
& \leq\left(20+\frac{118\left(N^{e}\right)^{2} C_{\mathrm{inv}}}{t o l}\right)|u|_{a, \Omega}^{2} \cdot
\end{aligned}
$$

From Lemma 6.4, we directly obtain a condition number estimate for the preconditioned system.

THEOREM 6.5. The condition number of the ACMS two-level Schwarz operator is bounded by

$$
\kappa\left(M_{\mathrm{ACMS}}^{-1} A\right) \leq\left(20+\frac{118\left(N^{e}\right)^{2} C_{\mathrm{inv}}}{t o l}\right)\left(\hat{N}_{c}+1\right) .
$$

The constant $\hat{N}_{c}$ is an upper bound for the number of overlapping subdomains each point $x \in \Omega$ may belong to. All constants are independent of $H, h$, and the contrast of the coefficient function $A$.

REMARK 6.6. Let us note that the condition number estimate is qualitatively similar to condition number estimates for adaptive FETI-DP in two dimensions; see [31, Theorem 4.16, Theorem 5.1]. 


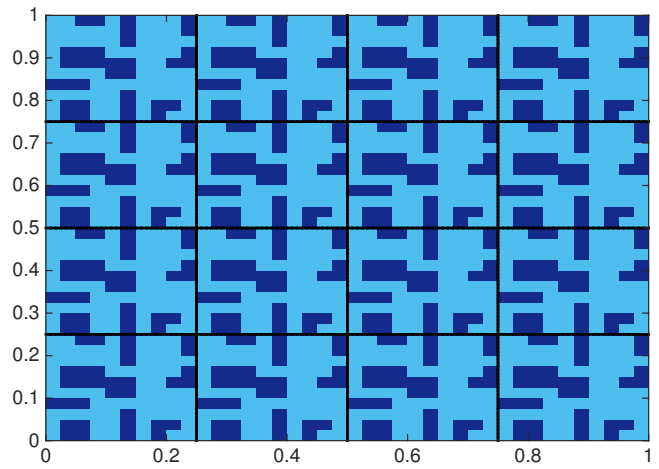

FIG. 7.1. Discontinuous coefficient function A with different types of channels and inclusions intersecting the interface. The light blue color corresponds to a coefficient of $A_{\min }=1.0$ and the dark blue color to a high coefficient of $A_{\max } ; 1 / H=4$.

TABLE 7.1

Numerical results for the coefficient function in Figure 7.1 with varying contrast $A_{\max } / A_{\min }$ : tolerance for the selection of the eigenfunctions, iteration counts, condition numbers, and resulting coarse space dimension for different coarse space variants; $1 / H=4, H / h=30$, and $\delta=2 h$. The new theory presented in this paper covers the two coarse spaces marked in bold face.

\begin{tabular}{|c|c|c|c|c|c|c|c|c|c|c|c|c|}
\hline \multirow{3}{*}{$\begin{array}{l}V_{0} \\
V_{\text {MsFEM }} \\
\end{array}$} & \multicolumn{4}{|c|}{$A_{\max }=10^{4}$} & \multicolumn{4}{|c|}{$A_{\max }=10^{6}$} & \multicolumn{4}{|c|}{$A_{\max }=10^{8}$} \\
\hline & \multirow{2}{*}{$\frac{t o l}{-}$} & \multirow{2}{*}{$\begin{array}{r}\text { it. } \\
134\end{array}$} & \multicolumn{2}{|c|}{$\kappa \operatorname{dim} V_{0}$} & \multirow{2}{*}{ tol } & \multirow{2}{*}{$\begin{array}{r}\text { it. } \\
196\end{array}$} & \multicolumn{2}{|c|}{$\kappa \operatorname{dim} V_{0}$} & \multirow{2}{*}{$\begin{array}{r}\text { tol } \\
-\end{array}$} & \multirow{2}{*}{$\begin{array}{r}\text { it. } \\
276\end{array}$} & \multicolumn{2}{|c|}{$\kappa \operatorname{dim} V_{0}$} \\
\hline & & & $7.83 \cdot 10^{3}$ & 9 & & & $7.82 \cdot 10^{5}$ & 9 & & & $7.82 \cdot 10^{7}$ & 9 \\
\hline$V_{\text {ACMS-D }}^{t o l}$ & $10^{-1}$ & 21 & 4.74 & 69 & $10^{-1}$ & 22 & 4.75 & 69 & $10^{-1}$ & 22 & 4.75 & 69 \\
\hline$V_{\text {ACMS- } \bar{D}}^{\text {tol }}$ & $10^{-1}$ & 21 & 4.74 & 69 & $10^{-1}$ & 22 & 4.75 & 69 & $10^{-1}$ & 22 & 4.75 & 69 \\
\hline $\mathrm{V}_{\text {ACMS-N }}^{\text {tol }}$ & $10^{-2}$ & 22 & 5.09 & 69 & $10^{-2}$ & 23 & 5.09 & 69 & $10^{-2}$ & 26 & 5.10 & 69 \\
\hline$V_{\text {ACMS- }-\bar{N}}^{\text {tol }}$ & $10^{-2}$ & 22 & 5.09 & 69 & $10^{-2}$ & 23 & 5.09 & 69 & $10^{-2}$ & 26 & 5.10 & 69 \\
\hline $\mathrm{V}_{\text {ACMS-N,1 }}^{\text {tol }}$ & $10^{-2}$ & 19 & 4.34 & 69 & $10^{-2}$ & 20 & 4.35 & 69 & $10^{-2}$ & 21 & 4.35 & 69 \\
\hline$V_{\text {ACMS- } \bar{N}, 1}^{t o l}$ & $10^{-2}$ & 19 & 4.34 & 69 & $10^{-2}$ & 20 & 4.35 & 69 & $10^{-2}$ & 21 & 4.35 & 69 \\
\hline$V_{\text {SHEM }}$ & $10^{-3}$ & 19 & 4.32 & 69 & $10^{-3}$ & 20 & 4.33 & 69 & $10^{-3}$ & 20 & 4.33 & 69 \\
\hline$V_{\text {ACMS-R }}^{\text {tol }}$ & 2.0 & 24 & 6.50 & 69 & 2.0 & 24 & 6.50 & 69 & 2.0 & 24 & 6.50 & 69 \\
\hline
\end{tabular}

Proof. Since we use exact local solvers, we directly obtain

$$
\kappa\left(M_{\mathrm{ACMS}}^{-1} A\right) \leq C_{0}^{2}\left(\hat{N}_{c}+1\right),
$$

where $C_{0}^{2}$ is the constant of the stable decomposition; cf. [40, Lemma 3.11] and the follow-up discussion and the proof of [12, Theorem 4.1]. We obtain the final estimate using Lemma 6.4. (

Let us note that in the numerical experiments considered in Section 7, we have $\hat{N}_{c}=4$.

7. Numerical results. We present numerical results which illustrate the robustness of our overlapping Schwarz method with the ACMS coarse space and which support our theory. Our numerical experiments also show that the dimension of our adaptive coarse space is usually small, i.e., our iterative method does not degenerate to a direct solver. This is important since, as in other domain decomposition methods with adaptive coarse spaces, the condition number is determined by the user, but the dimension of the resulting coarse space is determined by the algorithm and, in general, only known ex post.

In all numerical tests, we discretize (2.1) with $f \equiv 1$ using piecewise bilinear finite elements and solve the resulting linear system with the preconditioned conjugate gradient 

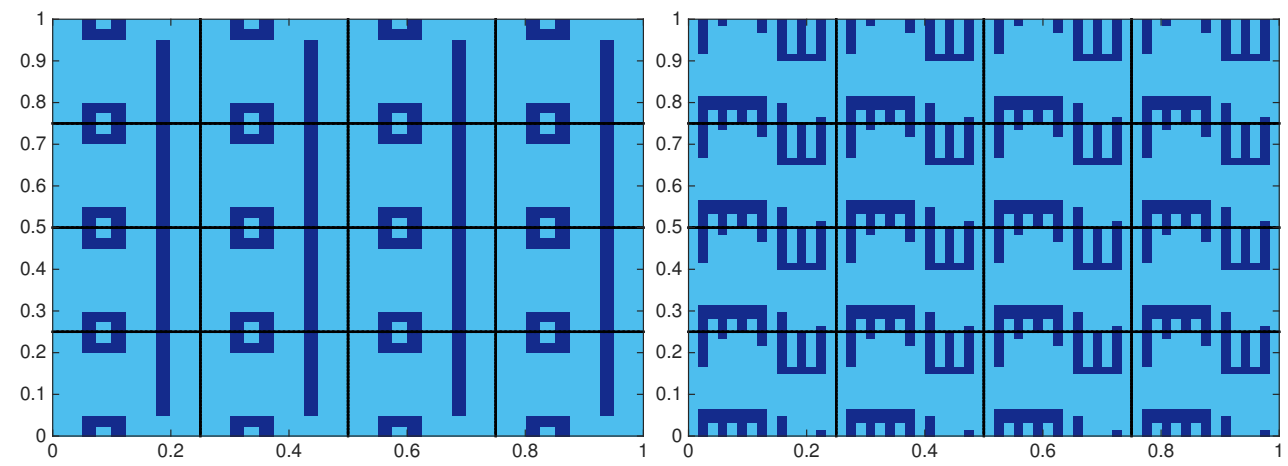

FIG. 7.2. Discontinuous coefficient functions A: circles and a channel intersecting several edges (left); combshaped inclusions intersecting the horizontal edges (right). The light blue color corresponds to a coefficient of $A_{\min }=1.0$ and the dark blue color to a high coefficient of $A_{\max } ; 1 / H=4$.

TABLE 7.2

Results for the coefficient functions in Figure 7.2: tolerance for the selection of the eigenfunctions, iteration counts, condition numbers, and resulting coarse space dimension for different coarse space variants; $1 / H=4$, $H / h=30$, and $\delta=2 h$; maximum coefficient $A_{\max }=10^{8}$.

\begin{tabular}{|c|c|c|c|c|c|c|c|c|}
\hline \multirow[b]{2}{*}{$V_{0}$} & \multicolumn{4}{|c|}{ Coeff. function $A$ from Figure 7.2 (left) } & \multicolumn{4}{|c|}{ Coeff. function $A$ from Figure 7.2 (right) } \\
\hline & tol & it. & $\kappa$ & & tol & it. & $\kappa$ & $\operatorname{dim} V_{0}$ \\
\hline$V_{\text {MsFEM }}$ & - & 313 & $1.36 \cdot 10^{8}$ & 9 & $\begin{array}{cc}- \\
-\end{array}$ & 281 & $3.77 \cdot 10^{7}$ & 9 \\
\hline$V_{\text {ACMS-D }}^{t o l}$ & $2 \cdot 10^{-1}$ & 31 & 30.00 & 33 & $10^{-2}$ & 52 & 44.74 & 33 \\
\hline$V_{\mathrm{ACMS}-\overline{\mathrm{D}}}^{t o l}$ & $2 \cdot 10^{-1}$ & 38 & 30.00 & 33 & $10^{-1}$ & 52 & 44.74 & 33 \\
\hline $\mathrm{V}_{\text {ACMS-N }}^{\text {tol }}$ & $10^{-2}$ & 27 & 7.14 & 33 & $10^{-2}$ & 41 & 13.22 & 33 \\
\hline$V_{\text {AcMS }-\bar{N}}^{\text {tol }}$ & $10^{-2}$ & 27 & 7.14 & 33 & $10^{-2}$ & 41 & 13.22 & 33 \\
\hline $\mathrm{V}_{\text {ACMS-N,1 }}^{\text {tol }}$ & $10^{-2}$ & 30 & 6.91 & 45 & $10^{-2}$ & 29 & 6.47 & 93 \\
\hline$V_{\text {ACMS- } \bar{N}, 1}^{\text {tol }}$ & $10^{-2}$ & 30 & 6.91 & 45 & $10^{-2}$ & 29 & 6.47 & 93 \\
\hline$V_{\text {SHEM }}$ & $10^{-3}$ & 28 & 6.92 & 45 & $10^{-3}$ & 29 & 6.39 & 93 \\
\hline$V_{\text {ACMS-R }}^{t o l}$ & 2.0 & 33 & 8.33 & 45 & 2.0 & 30 & 6.58 & 93 \\
\hline
\end{tabular}

method and a relative stopping criterion, $\left\|r^{(k)}\right\|_{2} /\left\|r^{(0)}\right\|_{2}<10^{-8}$, where $r^{(0)}$ and $r^{(k)}$ are the initial and $k$-th unpreconditioned residuals, respectively.

We consider the different variants of the ACMS coarse spaces in the numerical experiments. By $V_{\mathrm{ACMS}-\mathrm{D}}^{\text {tol }}$, we denote the method from Section 4.1, where Dirichlet boundary conditions are used. By $V_{\mathrm{ACMS}-\mathrm{N}}^{\text {tol }}$, we denote the method described in Section 4.2, where Neumann boundary conditions are used. In both cases tol is the user-defined tolerance. If mass lumping is used, then we denote the space by $V_{\mathrm{ACMS}-\overline{\mathrm{D}}}^{\text {tol }}$ or $V_{\mathrm{ACMS}-\overline{\mathrm{N}}}^{\text {tol }}$, respectively.

For the Neumann versions, we also consider variants using harmonic extensions only on slabs of width $h$; see Section 4.2. These are denoted as $V_{\mathrm{ACMS}-\mathrm{N}, 1}^{\text {tol }}$ or, when lumping the mass matrix, as $V_{\mathrm{ACMS}-\overline{\mathrm{N}}, 1}^{\text {tol }}$.

In the method $V_{\mathrm{ACMS}-\mathrm{N}, 1}^{\text {tol }}$ (cf. Section 4.2), the left-hand side of the eigenvalue problem is assembled using discrete harmonic functions supported only on narrow slabs of width $h$ (cf. Figure 4.1), and in the methods $V_{\mathrm{SHEM}}^{\text {tol }}$ and $V_{\mathrm{ACMS}-\mathrm{R}}^{\text {tol }}$, the work for the assembly and construction of the left-hand sides of the eigenvalue problems corresponds only to the degrees of freedom on the open edge. Therefore, we denote these four approaches as "economic". Let us note that the support of the right-hand side in all eigenvalue problems never exceeds a slab of width $h$ and is therefore not affected.

In the non-economic approaches (i.e., $V_{\mathrm{ACMS}-\mathrm{N}}^{\text {tol }}, V_{\mathrm{ACMS}-\overline{\mathrm{N}}}^{\text {tol }}, V_{\mathrm{ACMS}-\mathrm{D}}^{\text {tol }}$, and $V_{\mathrm{ACMS}-\overline{\mathrm{D}}}^{\text {tol }}$ ), 


\section{ETNA}

Kent State University and

Johann Radon Institute (RICAM)

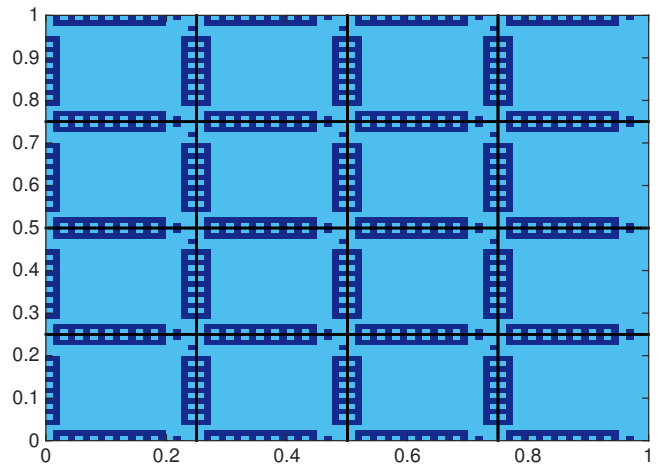

FIG. 7.3. Discontinuous coefficient function A with many connected channels intersecting the interface. The light blue color corresponds to a coefficient of $A_{\min }=1.0$ and the dark blue color to a high coefficient of $A_{\max }$; $1 / H=4$.

TABLE 7.3

Results for the coefficient functions in Figure 7.3: tolerance for the selection of the eigenfunctions, iteration counts, condition numbers, and resulting coarse space dimension for different coarse space variants; $1 / H=4$, $H / h=40$, and $\delta=2 h$; maximum coefficient $A_{\max }=10^{8}$.

\begin{tabular}{|c|c|c|c|c|}
\hline \multirow[b]{2}{*}{$V_{0}$} & \multicolumn{4}{|c|}{ Coeff. function $A$ from Figure 7.3} \\
\hline & tol & it. & $\kappa$ & $\operatorname{dim} V_{0}$ \\
\hline$V_{\mathrm{MsFEM}}$ & & 463 & $1.94 \cdot 10^{8}$ & 9 \\
\hline$V_{\mathrm{ACMS}-\mathrm{D}}^{\text {tol }}$ & $10^{-2}$ & 45 & 23.02 & 57 \\
\hline$V_{\mathrm{ACMS}-\overline{\mathrm{D}}}^{\text {tol }}$ & $10^{-2}$ & 45 & 23.02 & 57 \\
\hline $\mathrm{V}_{\text {ACMS-N }}^{\text {tol }}$ & $10^{-2}$ & 40 & 25.94 & 57 \\
\hline$V_{\mathrm{ACMS}-\overline{\mathrm{N}}}^{\text {tol }}$ & $10^{-2}$ & 40 & 25.94 & 57 \\
\hline $\mathrm{V}_{\mathrm{ACMS}-\mathrm{N}, \mathbf{1}}^{\text {tol }}$ & $10^{-2}$ & 21 & 5.17 & 213 \\
\hline$V_{\mathrm{ACMS}-\overline{\mathrm{N}}, 1}^{\text {tol }}$ & $10^{-2}$ & 21 & 5.17 & 213 \\
\hline$V_{\text {SHEM }}$ & $10^{-3}$ & 23 & 5.03 & 213 \\
\hline$V_{\mathrm{ACMS}-\mathrm{R}}^{t o l}$ & 2.0 & 26 & 6.22 & 213 \\
\hline
\end{tabular}

harmonic extensions to the complete subdomains are used leading to a higher computational cost. However, the use of the coefficient information on the complete subdomain can be beneficial: the resulting coarse space can be significantly smaller; see Table 7.2.

In all tables, we highlight in bold face the methods $\mathbf{V}_{\mathbf{A C M S}-\mathbf{N}}^{\text {tol }}$ and $\mathbf{V}_{\mathbf{A C M S}-\mathbf{N}, \mathbf{1}}^{\text {tol }}$, which are supported by our theory; see Section 6.

As described in Section 4.3, all methods which are denoted by a bar, i.e., $V_{\mathrm{ACMS}-\overline{\mathrm{D}}}^{\text {tol }}$, $V_{\mathrm{ACMS}-\overline{\mathrm{N}}}^{\text {tol }}$, and $V_{\mathrm{ACMS}-\overline{\mathrm{N}}, 1}^{\text {tol }}$, use a lumped mass matrix. Here, the eigenvalue problems can be transformed into standard eigenvalue problems with small computational effort, which leads to another reduction of computational work; this holds true also for the SHEM coarse space. However, we observe that lumping the mass matrix does not significantly affect the performance of the methods; cf. Tables 7.1, 7.2, 7.3, and 7.4.

In Table 7.1, for the coefficient function illustrated in Figure 7.1, we compare the different approaches of adaptive coarse spaces. We observe that for this problem the MsFEM coarse space $V_{\text {MsFEM }}$ is not sufficient: the condition number and the number of iterations is large since the MsFEM coarse space cannot cope with several heterogeneities intersecting an edge. For all other methods, a small condition number $\kappa$ (below 10) of the preconditioned operator can be obtained (if the tolerance $t o l$ is chosen appropriately) resulting in a number of approximately 20 conjugate gradient iterations. We also observe that all methods are robust 


\section{ETNA}

Kent State University and

Johann Radon Institute (RICAM)

TABLE 7.4

Results for the coefficient function in Figure 7.1: iteration counts, condition numbers, and resulting coarse space dimension for different coarse space variants; $1 / H=4, H / h=30$, and $\delta=2 h$; maximum coefficient $A_{\max }=10^{8}$.

\begin{tabular}{|c|c|c|c|c|c|c|}
\hline \multirow[b]{2}{*}{$V_{0}$} & \multicolumn{3}{|c|}{1 LobPCG it. } & \multicolumn{3}{|c|}{ Direct eigensolver } \\
\hline & it. & $\kappa$ & $\operatorname{dim} V_{0}$ & it. & $\kappa$ & $\operatorname{dim} V_{0}$ \\
\hline$V_{\mathrm{ACMS}-\mathrm{D}}^{10^{-2}}$ & 22 & 4.75 & 69 & 22 & 4.75 & 69 \\
\hline$V_{\mathrm{ACMS}-\overline{\mathrm{D}}}^{10^{-1}}$ & 22 & 4.75 & 69 & 22 & 4.75 & 69 \\
\hline $\mathrm{V}_{\mathrm{ACMS}-\mathrm{N}}^{10^{-2}}$ & 23 & 5.09 & 69 & 23 & 5.09 & 69 \\
\hline$V_{\mathrm{ACMS}-\overline{\mathrm{N}}}^{10^{-2}}$ & 23 & 5.09 & 69 & 23 & 5.09 & 69 \\
\hline $\mathrm{V}_{\mathrm{ACMS}-\mathrm{N}, 1}^{10^{-2}}$ & 20 & 4.35 & 69 & 20 & 4.35 & 69 \\
\hline$V_{\mathrm{ACMS}-\overline{\mathrm{N}}, 1}^{10^{-2}}$ & 20 & 4.35 & 69 & 20 & 4.35 & 69 \\
\hline$V_{\text {SHEM }}$ & 20 & 4.33 & 69 & 20 & 4.33 & 69 \\
\hline
\end{tabular}

with respect to variations in the contrast of the coefficient function. Note that the size of the coarse space is identical in all cases of adaptive coarse spaces.

At first sight, the results are similar for the coefficient functions depicted in Figure 7.2 (left and right); see Table 7.2. However, now the dimensions of the coarse spaces differ. In the methods where the construction of the eigenvalue problem is economic, the coarse space is larger. The reason is that, e.g., for the ring-like structure in Figure 7.2 (left), these methods cannot detect that the ring is a connected structure and thus a single eigenvector is sufficient. This is even more pronounced for the comb-like structures in Figure 7.2 (right).

To investigate this effect further, we have considered the coefficient function in Figure 7.3, where on each edge a comb-like structure is placed in addition to a small inclusion. Here, for each edge, the non-economic coarse spaces adds two eigenvalues to the coarse space resulting in a dimension of $9+48=57$. On the other hand, for the economic versions (which includes $V_{\text {SHEM }}$ ), for each horizontal and vertical edge, 9 and 8 eigenvectors are used, respectively. As a result, the coarse space is larger by a factor of almost 4 for the economic versions; see Table 7.3. It is clear that for an (artificial) example of a very fine comb on a very fine grid the coarse space constructed from the economic versions can be larger by an arbitrary factor compared to our coarse spaces $V_{\mathrm{ACMS}-\mathrm{D}}^{t o l}$ and $V_{\mathrm{ACMS}-\mathrm{N}}^{t o l}$ (or the corresponding lumped versions $V_{\mathrm{ACMS}-\overline{\mathrm{D}}}^{t o l}$ and $V_{\mathrm{ACMS}-\overline{\mathrm{N}} \text { ). }}^{\text {tol }}$.

It is clear that the solution of the eigenvalue problems can be costly. First, note that we need to solve only standard eigenvalue problems instead of generalized eigenvalue problems. In particular, for the versions with a lumped mass matrix this is computationally inexpensive; see Section 4.3.

To show how the computational cost can be reduced further, in Table 7.4, we present numerical experiments where the eigenvalue problems are solved approximately by using only one iteration of LobPCG [32] using the Cholesky decomposition of the Schur complement matrix as a preconditioner: instead of the eigenfunctions corresponding to the smallest eigenvalues of (4.9), we solve for the smallest eigenvalues of

$$
-B_{e_{i j}} V=\lambda S_{e_{i j}} V
$$

and use the inverse of $S_{e_{i j}}$ as the preconditioner in the LobPCG iteration. The initial vectors were generated randomly using a normal distribution, and then one step of the power iteration for $S_{e_{i j}}^{-1}$ was applied to obtain a better initial guess. Note that this step only requires one additional forward-backward substitution since the Cholesky decomposition of $S_{e_{i j}}$ computed before the LobPCG iteration can be used. A block size of 3 was chosen, i.e., a block of three eigenvector approximations is computed in each LobPCG iteration. The results show that 
a single iteration of LobPCG is sufficient and gives the same results as a direct eigensolver (LAPACK) for the coefficient function in Figure 7.1; see Table 7.4. The cost for approximating the eigenvectors thus is comparable to a Cholesky decomposition of the Schur complement $S_{e_{i j}}$ on the edge. Let use note that LobPCG as an eigensolver for the computation of the adaptive coarse spaces in FETI-DP has been already successfully used in [29].

In some methods, the choice of the tolerance tol is more difficult than in others. In the methods using Neumann boundary conditions (i.e., $V_{\mathrm{ACMS}-\mathrm{N}}^{\text {tol }}, V_{\mathrm{ACMS}-\overline{\mathrm{N}}}^{\text {tol }}, V_{\mathrm{ACMS}-\mathrm{N}, 1}^{\text {tol }}$, $\left.V_{\mathrm{ACMS}-\overline{\mathrm{N}}, 1}^{\text {tol }}, V_{\mathrm{SHEM}}\right)$ there is a clear spectral gap, and we observe that the tolerance $t o l$ can be chosen relatively easy for different values of $H / h$; in our experiments, we can indeed use $t o l=10^{-2}$ for all our computations. However, for the methods using Dirichlet conditions, the choice is more difficult since the gap in the spectrum is smaller.

Let us note that currently, in the construction of the adaptive coarse space, we have not taken advantage of the fact that the MsFEM space also removes certain bad eigenvalues. Therefore, in the best case, the size of our coarse space could be reduced by one for each edge with a heterogeneity.

The method described here for overlapping domain decompositions is related (as well as the other approaches $[11,16,17,19])$ to the approach in [30] for the FETI-DP and BDDC methods, where also a Poincaré inequality is replaced by an eigenvalue problem. However, for nonoverlapping domain decomposition, to obtain robustness for certain cases, an additional eigenvalue problem is needed to replace an extension theorem.

8. Conclusion. We have introduced an overlapping Schwarz method using an ACMSbased coarse space. This coarse space uses eigenvalue problems on edges. We then have shown that the condition number of the method can be controlled independently of the heterogeneities in the problem, and, finally, we have provided supporting numerical experiments. We have observed that our coarse space can be smaller by a large factor compared to other competing coarse spaces for special coefficient functions while still remaining computationally inexpensive. Indeed, we have also shown numerically that the approximation of the local eigenvectors by a single iteration of preconditioned LobPCG may be sufficient. Among the competing approaches that generate small coarse spaces, to the best of our knowledge, our approach uses eigenvalue problems with the smallest dimension.

\section{REFERENCES}

[1] J. AARNES AND T. Y. Hou, Multiscale domain decomposition methods for elliptic problems with high aspect ratios, Acta Math. Appl. Sin. Engl. Ser., 18 (2002), pp. 63-76.

[2] I. BABUŠKA AND J. E. OSBORN, Generalized finite element methods: their performance and their relation to mixed methods, SIAM J. Numer. Anal., 20 (1983), pp. 510-536.

[3] I. BABUŠKA, U. BANERJEE, AND J. E. OSBORN, Generalized finite element methods-main ideas, results and perspective, Int. J. Comput. Methods, 1 (2004), pp. 67-103.

[4] P. BJøRSTAD, J. Koster, AND P. KRZYZANOWSKi, Domain decomposition solvers for large scale industrial finite element problems, in Applied Parallel Computing. New Paradigms for HPC in Industry and Academia, T. Sørevik, F. Manne, A. H. Gebremedhin, and R. Moe, eds., vol. 1947 of Lect. Notes Comput. Sci., Springer, Berlin, 2001, pp. 373-383.

[5] M. Buck, Overlapping Domain Decomposition Preconditioners for Multi-Phase Elastic Composites, $\mathrm{PhD}$. Thesis, Fachbereich Mathematik, Technische Universität Kaiserslautern, Kaiserslautern, 2013.

[6] M. BUCK, O. ILIEV, AND H. ANDRÄ, Multiscale finite elements for linear elasticity: oscillatory boundary conditions, in Domain Decomposition Methods in Science and Engineering XXI, J. Erhel, M. J. Gander, L. Halpern, G. Pichot, T. Sassi, and O. Widlund, eds., vol. 98 of Lect. Notes Comput. Sci. Eng., Springer, Cham, 2014, pp. 237-245.

[7] R. R. CRAIG AND M. C. BAMPTON, Coupling of substructures for dynamic analyses, AIAA Journal, 6 (1968), pp. 1313-1319.

[8] C. R. Dohrmann, A. Klawonn, and O. B. Widlund, Domain decomposition for less regular subdomains: overlapping Schwarz in two dimensions, SIAM J. Numer. Anal., 46 (2008), pp. 2153-2168. 
[9] C. R. Dohrmann AND O. B. Widlund, Hybrid domain decomposition algorithms for compressible and almost incompressible elasticity, Internat. J. Numer. Methods Engrg., 82 (2010), pp. 157-183.

[10] - Lower dimensional coarse spaces for domain decomposition, in Domain Decomposition Methods in Science and Engineering XXI, J. Erhel, M. J. Gander, L. Halpern, G. Pichot, T. Sassi, and O. Widlund, eds., vol. 98 of Lect. Notes Comput. Sci. Eng., Springer, Cham, 2014, pp. 527-535.

[11] V. Dolean, F. Nataf, R. Scheichl, AND N. SPILlane, Analysis of a two-level Schwarz method with coarse spaces based on local Dirichlet-to-Neumann maps, Comput. Methods Appl. Math., 12 (2012), pp. 391-414.

[12] M. DRYJA AND O. B. WIDlund, Domain decomposition algorithms with small overlap, SIAM J. Sci. Comput., 15 (1994), pp. 604-620.

[13] W. E AND B. EngQuist, The heterogeneous multiscale methods, Commun. Math. Sci., 1 (2003), pp. 87-132.

[14] W. E, B. ENGQUist, X. LI, W. Ren, AND E. VANDEN-EIJNDEN, Heterogeneous multiscale method: a review, Commun. Comput. Phys., 2 (2007), pp. 367-450.

[15] Y. Efendiev AND T. Y. Hou, Multiscale Finite Element Methods. Theory and Applications, Springer, New York, 2009.

[16] J. GALVIS AND Y. EFENDIEv, Domain decomposition preconditioners for multiscale flows in high-contrast media, Multiscale Model. Simul., 8 (2010), pp. 1461-1483.

[17] _ Domain decomposition preconditioners for multiscale flows in high contrast media: reduced dimension coarse spaces, Multiscale Model. Simul., 8 (2010), pp. 1621-1644.

[18] M. J. GANDER AND A. LONELAND, SHEM: an optimal coarse space for RAS and its multiscale approximation, in Domain Decomposition Methods in Science and Engineering XXIII, C.-O. Lee, X.-C. Cai, D. E. Keyes, H. H. Kim, A. Klawonn, E.-J. Park, and O. B. Widlund, vol. 116 of Lect. Notes Comput. Sci. Eng., Springer, Cham, 2017, pp. 313-321.

[19] M. J. Gander, A. Loneland, AND T. Rahman, Analysis of a new harmonically enriched multiscale coarse space for domain decompostion methods, Preprint on arXiv, 2015. https://arxiv.org/abs/1512.05285

[20] I. G. GRAham, P. O. LeChNER, AND R. SCHEICHL, Domain decomposition for multiscale PDEs, Numer. Math., 106 (2007), pp. 589-626.

[21] A. HeInLEIN, Parallel Overlapping Schwarz Preconditioners and Multiscale Discretizations with Applications to Fluid-Structure Interaction and Highly Heterogeneous Problems, PhD. Thesis, Math.-NaWi. Fakultät, University of Cologne, Cologne, 2016.

[22] A. Heinlein, U. Hetmaniuk, A. Klawonn, and O. Rheinbach, The approximate component mode synthesis special finite element method in two dimensions: parallel implementation and numerical results, J. Comput. Appl. Math., 289 (2015), pp. 116-133.

[23] A. Heinlein, A. KlawONn, AND O. RHEINBACH, A parallel implementation of a two-level overlapping Schwarz method with energy-minimizing coarse space based on Trilinos, SIAM J. Sci. Comput., 38 (2016), pp. C713-C747.

[24] U. HetMANIUK AND A. Klawonn, Error estimates for a two-dimensional special finite element method based on component mode synthesis, Electron. Trans. Numer. Anal., 41 (2014), pp. 109-132. http://etna.ricam.oeaw.ac.at/vol.41.2014/pp109-132.dir/pp109-132.pdf

[25] U. L. HETMANIUK AND R. B. LeHOUCQ, A special finite element method based on component mode synthesis, M2AN Math. Model. Numer. Anal., 44 (2010), pp. 401-420.

[26] T. Y. HoU AND X.-H. WU, A multiscale finite element method for elliptic problems in composite materials and porous media, J. Comput. Phys., 134 (1997), pp. 169-189.

[27] W. C. HURTY, Vibrations of structural systems by component-mode synthesis, J. Engrg. Mech. Div., 86 (1960), pp. 51-69.

[28] H. H. KIM, E. CHUNG, AND J. WANG, BDDC and FETI-DP preconditioners with adaptive coarse spaces for three-dimensional elliptic problems with oscillatory and high contrast coefficients, J. Comput. Phys., 349 (2017), pp. 191-214.

[29] A. Klawonn, M. KÜHn, AND O. RheInBACH, Adaptive coarse spaces for FETI-DP in three dimensions, SIAM J. Sci. Comput., 38 (2016), pp. A2880-A2911.

[30] A. KLAWONn, P. RADTKE, AND O. RHEINBACH, FETI-DP methods with an adaptive coarse space, SIAM J. Numer. Anal., 53 (2015), pp. 297-320.

[31] _ A comparison of adaptive coarse spaces for iterative substructuring in two dimensions, Electron. Trans. Numer. Anal., 45 (2016), pp. 75-106. http://etna.ricam.oeaw.ac.at/vol.45.2016/pp75-106.dir/pp75-106.pdf

[32] A. V. KNYAZEV, Toward the optimal preconditioned eigensolver: locally optimal block preconditioned conjugate gradient method, SIAM J. Sci. Comput., 23 (2001), pp. 517-541.

[33] J. MANDEL AND B. SousedíK, Adaptive selection of face coarse degrees of freedom in the BDDC and the FETI-DP iterative substructuring methods, Comput. Methods Appl. Mech. Engrg., 196 (2007), pp. 1389-1399. 
[34] J. Mandel, B. Sousedík, And J. Šístek, Adaptive BDDC in three dimensions, Math. Comput. Simulation, 82 (2012), pp. 1812-1831.

[35] J. Nolen, G. Papanicolaou, and O. Pironneau, A framework for adaptive multiscale methods for elliptic problems, Multiscale Model. Simul., 7 (2008), pp. 171-196.

[36] C. Pechstein And C. R. Dohrmann, Modern domain decomposition methods, BDDC, deluxe scaling, and an algebraic approach, Talk by C. Pechstein at the University Linz, December 2013.

http://people.ricam.oeaw.ac.at/c.pechstein/pechstein-bddc2013.pdf

[37] - A unified framework for adaptive BDDC, Electron. Trans. Numer. Anal., 46 (2017), pp. 273-336. http://etna.ricam.oeaw.ac.at/vol.46.2017/pp273-336.dir/pp273-336.pdf

[38] N. Spillane, V. Dolean, P. Hauret, F. Nataf, C. Pechstein, and R. Scheichl, Abstract robust coarse spaces for systems of PDEs via generalized eigenproblems in the overlaps, Numer. Math., 126 (2014), pp. 741-770.

[39] N. SPILlane AND D. J. RiXen, Automatic spectral coarse spaces for robust finite element tearing and interconnecting and balanced domain decomposition algorithms, Internat. J. Numer. Methods Engrg., 95 (2013), pp. 953-990.

[40] A. Toselli And O. Widlund, Domain Decomposition Methods-Algorithms and Theory, Springer, Berlin, 2005.

[41] R. VERFÜRTH, A Posteriori Error Estimation Techniques for Finite Element Methods, Oxford University Press, Oxford, 2013. 